\title{
Spheroidally deformed sodium clusters in the selfconsistent jellium model
}

\author{
Th. Hirschmann ${ }^{1}$, M. Brack ${ }^{1}$, and J. Meyer $^{2}$ \\ ${ }^{1}$ Institut für Theoretische Physik, Universität Regensburg, D-93040 Regensburg, Germany \\ ${ }^{2}$ Institut de Physique Nucléaire (et IN2P3), Université de Lyon-I, F-69622 Villeurbanne, France
}

Received 1 December 1993, revised version 2 February 1994, accepted 28 April 1994

\begin{abstract}
We present the first systematic study of potential energy curves and prolate-oblate shape transitions of sodium clusters with $8<N<40$ atoms. The Kohn-Sham equations are solved in the local density approximation for the jellium model with spheroidal deformations. The ionic background density is taken to have a diffuse surface of Woods-Saxon type. The quadrupole and hexadecupole moments of the electron and jellium densities are investigated, revealing a strong hexadecupole dependence for selected clusters. Collective dipole resonances are described in the simple surface plasmon model. Shape transitions are found to occur at particle numbers 12-14 (prolate-oblate), 18-20-22 (oblate-spherical-prolate) and 30-32 (prolate-oblate), which are in good agreement with experimental results; triaxiality is predicted for $\mathrm{Na}-36$. Comparing our results with those of molecular dynamics calculations, we confirm the scheme of Kohn-Sham levels and the gross behaviour of potentials and densities.
\end{abstract}

Keywords: Metal clusters; Spheroidal jellium model; Deformation energy.

\section{Introduction}

The selfconsistent spherical jellium model for finite clusters, introduced in the ThomasFermi approximation by Cini [1] and in the microscopic form independently by Hintermann and Manninen [2], Ekardt [3] and by Beck [4], has been quite successful for a semi-quantitative description of many experimental properties of alkali metal clusters. The most prominent of them is the existence of peaks in the mass abundances obtained from cluster beams, which is explained in terms of the 'magic numbers' $8,20,40,58$, $92, \ldots$, corresponding to filled spherical (main) shells. But also the average trends and general size dependence of ionization potentials and electron affinities, dipole polarizabilities, and the energies of resonance peaks in photoabsorption spectra are well described in the jellium model. (For recent reviews, see e.g. de Heer [5] and Brack [6].)

In alkali and some other simple metals, such as $\mathrm{Cu}$ and $\mathrm{Ag}$, the strong delocalization of the valence electrons - in particular if they have $s$-wave character - allows one to ignore in a first approach the ionic structure of the clusters and to treat the valence electrons in a selfconsistent mean-field approach in which the ionic charges enter only in the form of a homogeneous background charge density: the so-called jellium density. The quantization of single-particle states in a finite volume, together with the Pauli 
principle, then leads directly to the existence of electronic shell structure in most cluster observables and to the formulation of simple phenomenological shell models for clusters [7-9].

This simple approach is most likely justified in large systems where the valence electrons form a quasi-free gas confined by the cluster surface, their Fermi wave length being much larger than the average ionic bond lengths. It is, indeed, strongly supported by the quantitative explanation of the so-called 'supershell' structure [9], which has been experimentally observed [10-12] and well reproduced in the selfconsistent jellium model [13], in terms of closed classical orbits of the electrons in a spherical cavity [14] or a Woods-Saxon-like potential with steep walls [9].

Surprisingly, however, the jellium model reproduces the above-mentioned cluster properties quite well also in small systems with even less than 20 atoms, where quantum-chemical methods (see e.g. [15] for a recent review) and molecular dynamics calculations (see e.g. [16] or [17]) show that the ionic structure is not negligible and its inclusion leads to a more quantitative description of the observed data. In spite of its shortcomings and limitations and by virtue of its computational simplicity, the jellium model can therefore be used also for smaller clusters - at least to gain a first classification of cluster data and their size dependence.

This article is devoted to the deformation properties of sodium clusters with $8<N<40$ atoms. It was, indeed, realized early in the interpretation of the sodium cluster mass spectra of Knight et al. [18], that the fine structure between the main peaks corresponding to spherical closed shells is due to the static deformation of these clusters in their ground states. Clemenger [7] successfully adapted the well-known Nilsson model [19] from nuclear physics to the cluster data and could explain the minor peaks by the filling of deformed sub-shells. The appearance of static deformation is a consequence of the Jahn-Teller effect [20]: when a spherical shell with its $2(2 l+1)$-fold degeneracy (including spin) is only partially filled, the ground state of the cluster is degenerate; the system lifts this degeneracy by allowing the mean field to be deformed, thus spontaneously breaking its spherical symmetry, which results in a gain in total binding energy. This is also observed in the selfconsistent jellium model, as shown by Ekardt and Penzar [21], who solved the Kohn-Sham equations for axially deformed sodium clusters in spheroidal coordinates and confirmed the existence of deformed equilibrium shapes in the size regions $8<N<20$ and $20<N<40$.

More recently, Lauritsch et al. [22] performed similar calculations for clusters with $N<20$ without axial symmetry and found $\mathrm{Na}-12$ and $\mathrm{Na}-16$ to be triaxial, in agreement with phenomenological shell model predictions [23].

The main assumption of the 'deformed jellium model' is that the shape of the positive background density adapts itself to that of the electronic density distribution, which suggests itself from the large ductility of alkali metals. Practically, one obtains the ground state of a cluster by minimizing the total binding energy with respect to the deformation (or the overall shape) of the jellium density. This is very much in line with the Born-Oppenheimer approximation used both in quantum chemistry and molecular dynamics: The ionic distribution is determined adiabatically by the energy minimization principle, assuming that the electrons adjust themselves instantaneously to the external ionic potential.

The results of the static deformed jellium model calculations [21, 22, 24] are on the average well supported by those of quantum-chemical ab initio [15] and molecular dynamics (MD) calculations [17] where the ionic structure is fully taken into account. One can say that the jellium model is able to reproduce the averaged spatial distribution of 
the ions in terms of ellipsoidal shapes or, more generally, of the lowest-order multipole moments of the ionic density. In view of the huge numerical efforts required by those structural models, in particular if larger atomic numbers than $\sim 20$ are concerned, it seems worthwhile to pursue the jellium approach, especially in the direction of investigating the role of more refined cluster shapes and of more detailed comparisons of its predictions with those of $a b$ initio and MD calculations.

The present work constitutes an extension of the spheroidal model by Ekardt and Penzar [21]. Whereas our way of solving the Kohn-Sham equations for spheroidally deformed clusters in a deformed basis of single-particle wave functions is very similar to theirs, we shall present more detailed results of cluster deformation properties. More than just giving their ground state deformations in terms of one parameter $\delta$, we shall present deformation energy curves and multipole moments of both electron and jellium densities and discuss the systematics of shape isomerism. Some preliminary results of our program have been included in the work by Lauritsch et al. [22], where they were tested against the results of triaxial calculations using an altogether different numerical code, and some have been published in a conference contribution [25]. As a first step towards collective dynamics, we discuss the peak energies of the resonances in photoabsorption spectra, which now have been measured systematically in the considered mass range for sodium clusters, and interpret them in terms of the simple surface plasmon model based on RPA sum rules. As we shall show, the jellium model prediction for the shape transitions spherical-prolate-oblate-spherical in both mass regions $8 \leq N \leq 20$ and $20 \leq N \leq 40$ is well supported by the experiment. Finally, we shall compare our Kohn-Sham single-particle energies and the spherically-averaged electronic densities and mean fields with recent $\mathrm{MD}$ results.

\section{The jellium model for deformed metal clusters}

\subsection{Total energy and Kohn-Sham equations}

Using density functional theory, the total energy of a metal cluster in the spherical jellium model [3] is expressed as a functional of the local electronic single-particle density $\rho(\mathbf{r})$ :

$$
E[\rho]=T_{s}[\rho]+E_{H}+E_{e J}+E_{x c}[\rho]+E_{J},
$$

where

$$
\begin{aligned}
& E_{H}=\frac{e^{2}}{2} \iint d^{3} r d^{3} r^{\prime} \frac{\rho(\mathbf{r}) \rho\left(\mathbf{r}^{\prime}\right)}{\left|\mathbf{r}-\mathbf{r}^{\prime}\right|}, \\
& E_{e J}=\int d^{3} r \rho(\mathbf{r}) V_{J}(\mathbf{r}) .
\end{aligned}
$$

$T_{s}[\rho]$ is the kinetic energy for non-interacting particles with density $\rho(\mathbf{r}), E_{H}$ is the direct (Hartree) Coulomb energy of the electrons, and $E_{e J}$ the attractive electronjellium energy in terms of the external ionic potential $V_{J}$ discussed in the section below. $E_{x c}[\rho]$ is the exchange-correlation energy in the local density approximation which we take from Gunnarsson and Lundqvist [26]. $E_{J}$ is the self energy of the 
positive ionic charge which does not depend on $\rho$. Note that the sum of the repulsive electron-electron and ion-ion forces are almost cancelled by the electron-ion attraction. The binding of the valence electrons is therefore due to exchange and correlation effects, as is well-known for the binding in bulk metals.

The use of the local density approximation (LDA) for the exchange-correlation functional $E_{x c}[\rho]$ might be questioned for metallic clusters since their density drops vary rapidly in the surface region. One well-known shortcoming of the LDA is that the mean field (i.e., the Kohn-Sham potential) falls off faster than like $1 / r$ at large distances $r$ from the cluster. To remedy this defect, two extensions of the LDA are currently in use: i) a self-interaction correction (SIC), proposed by Perdew and Zunger [27], and ii) a weighted-density approximation (WDA) $[28,29]$ (we refer to the book by Dreizler and Gross [30] for a detailed discussion of these methods and to Gross and Kohn [31] for the extension of the SIC to the time-dependent LDA). Both methods have been applied to metal clusters with some success [32-36] (see the discussion in Sec. III.C of Ref. [6] for details). On the other hand, both i) Hartree-Fock calculations in which the exchange is treated exactly [37] and ii) the explicit evaluation of long-range correlations in the random phase approximation (RPA) $[37,38]$ have demonstrated that - at least within the spherical jellium model - the LDA works surprisingly well, even in small clusters, for the evaluation of bulk properties such as energies, polarizabilities and the positions of the collective dipole excitations. We therefore have chosen here to use the LDA in our study of deformation effects. The correct use of the SIC and WDA makes sense only in a spin density formalism which would give rise to further numerical complications due to the lack of time reversal invariance and to the necessity of solving two coupled Kohn-Sham equations.

The electron density $\rho(\mathbf{r})$ has to be normalized to the number of valence electrons which for neutral clusters of monovalent metals is equal to the number $N$ of atoms:

$$
\int \rho(\mathbf{r}) d^{3} r=N
$$

Writing the density in terms of single-particle wavefunctions $\phi_{i}(\mathbf{r})$

$$
\rho(\mathbf{r})=\sum_{i}\left|\phi_{i}(\mathbf{r})\right|^{2}
$$

the variation of the energy $E[\rho]$ with respect to $\phi_{i}^{*}(\mathbf{r})$ leads to the Kohn-Sham equations [39]:

$$
\left\{\hat{T}+V_{K S}(\mathbf{r})\right\} \phi_{i}(\mathbf{r})=\varepsilon_{i} \phi_{i}(\mathbf{r})
$$

The kinetic and the direct Coulomb part of the total energy are unique and exactly known functionals for all particle numbers. The only approximations lie in the external potential $V_{J}(\mathbf{r})$ derived from the ionic density and the exchange-correlation functional $E_{x c}[\rho]$.

\subsection{Jellium potential}

The external potential $V_{J}$ felt by the valence electrons is in general given by 


$$
V_{J}(\mathbf{r})=-e^{2} \int d^{3} r^{\prime} \frac{\rho_{J}\left(\mathbf{r}^{\prime}\right)}{\left|\mathbf{r}-\mathbf{r}^{\prime}\right|}
$$

in terms of the ionic density $\rho_{J}$. Different approaches are currently being investigated to take into account the ionic structure in the density distribution $\rho_{J}$.

The molecular dynamics method (MD) by Car and Parrinello [40] is a prominent way to include the ionic structure by solving Newton's equations of motion for the core atoms. The valence electron system and its interaction with the ions are determined by solving the Kohn-Sham equations at each time step. This is, of course, a very time consuming procedure. Furthermore, in alkali systems the delocalization of the $s$-electrons leads to a highly pronounced isomerism of almost degenerate minima, such that finding the ground state is numerically very involved, in particular for larger systems [17].

In the jellium model, the ionic structure is ignored altogether and the ionic density assumed to be constant inside the cluster and zero outside. For spherical clusters, $\rho_{J}(r)$ is simply a step function

$$
\rho_{J}(r)=\rho_{0} \Theta\left(r-R_{I}\right)
$$

with $\rho_{0}$ and $R_{I}$ chosen such that it is normalized to $N$ and reproduces the correct Wigner-Seitz radius $r_{s}$ of the bulk metal:

$$
R_{I}=r_{s} N^{1 / 3}, \rho_{0}=\left(4 \pi r_{s}^{3} / 3\right)^{-1}
$$

From this, it is a trivial matter to calculate the potential Eq. (6). For nonspherical clusters, the density $\rho_{J}$ can be taken to be a step function along a spheroidal [21] or any deformed surface; the potential $V_{J}$ must be obtained numerically in that case.

Although the jellium model is extremely crude with respect to the ionic charge distribution, several arguments are in favour of this simple approximation. First, the electrons at the Fermi surface, which are most important in determining the shell structure of finite clusters, have a de Broglie wave length which is typically three times larger than the average interionic distance. Second, the ionic potential - which in MD calculations is taken to be a more or less sophisticated pseudopotential - contributes only a relatively small perturbation to the delocalized $s$-electrons in alkali clusters. Finally, a finite temperature leads to a statistically averaged motion of the ions. Therefore the electrons only feel an averaged ionic potential inside the cluster. Even for zero temperature this holds because of the zero-point motion of the ions (which is not included in MD calculations due to the classical treatment of the ionic motion).

Due to all these arguments, the neglect of ionic structure in the interior of a cluster is a rather good lowest-order approximation. Nevertheless, there exist mainly two ways to include ionic effects in a simple way by means of averaging procedures:

i) Take into account the electrostatic energy difference of an ionic system with pointlike charges and a jellium system with a homogeneous charge distribution. UtrerasDiaz and Shore have shown that an additional inclusion of the electron-ion interaction within second-order perturbation theory reveals that the surface properties of the infinite metal only weakly depend on the position of the ions [41], and thus were able to repair the failure of the simple jellium model to reproduce the correct sign of the surface energy in the higher-density metals like aluminum [42]. The further development of these ideas led to the so-called 'stabilized jellium model' $[43,44]$ and more 
recently to a 'structure-averaged jellium model' [45]. These models make use of a variational principle for the Ashcroft empty-core radius $r_{c}$ within the framework of a liquid-drop expansion of the energy functional and allow for a better reproduction of the empirical surface energies of all metals. Moreover, it has been shown in these models $[43,45]$ that the central jellium density $\rho_{J}$ in finite clusters is different from the bulk value.

ii) Spherical averaging of the ionic pseudopotentials (SAPS) reduces the numerical effort to relax the ionic configuration by reducing the spatial dimension to one. The so-called 'SAPS model' [46] has been used by many groups; for a recent summary of its results we refer to Borstel et al. [47].

Obviously, the SAPS model does not lend itself to the study of deformation effects, whereas an extension of the structure-averaged jellium model to deformed clusters is in preparation [48].

We presently use, as a simple phenomenological lowest-order inclusion of ionic structure effects, the jellium model with a diffuse ionic density distribution $[22,25,49$, 50]. Indeed, the first place where one wants to improve the jellium model - without sacrificing its computational simplicity - is the cluster surface. This is where the valence electrons are reflected and their motion is quantized, leading to the electronic shell effects. Reinhard et al. [50] proposed to fold the step-like jellium density with a simple Ashcroft type pseudopotential [51] in order to include in a schematic way the influence of the ionic potentials on the cluster surface. This leads to a diffusivity of the ionic density distribution of the order of the pseudopotential core radius $r_{c}$ [51]. Its effect on the results of spherical jellium calculations are beneficial [50]: it increases the static dipole polarizabilities and decreases the energies of collective dipole oscillations approximately to their correct experimental average values. Furthermore it also improves the 'magicity' of the $N=40$ alkali clusters, as will be further discussed in Sect. 3, and decreases the average ionization potentials. Thus, the surface diffusivity of $\rho_{J}$ corrects several of the well-known deficiencies of the standard sharp-edged jellium model (see also Ref. [6]). We also point out that, although the sharp-edged jellium model has become quite standard and popular, we can think of no reason why, a priori, a smooth ionic density surface should be less physical than a step function.

The beneficial effects of a smooth jellium surface had already been observed by Rubio et al. [49], who used a parametrized density profile function for $\rho_{J}(r)$ and minimized the total energy of the cluster with respect to the diffusivity parameter, and by Lauritsch et al. [22] who used a simple Fermi function

$$
\rho_{J}(r)=\frac{\rho_{0}}{1+\exp \left[\left(r-R_{J}\right) / \alpha\right]}
$$

with a fixed value $\alpha=1$ a.u. (which is close to the values found by energy minimization $[49,50])$ and $R_{J}$ adjusted to conserve the number of ions.

In the present work we shall be using a diffuse jellium density distribution which for spherical clusters is given by Eq. (9) and generalized to deformed cluster shapes in the following. We should emphasize, however, that the diffuse jellium profile is not essential for most of the deformation properties discussed in this article, which are mainly determined by the overall shape of the spheroidal cluster as expressed by its axis ratio or its multipole moments. If we adhere to the diffuse jellium model here, it is mainly because of the above-mentioned improvements of experimental observables and of its numerical advantages in performing the Coulomb integrals over the deformed jellium density. 
The deformation of a sharp-edged spheroid can easily be expressed by the ratio $q$ of its semi-axes. Clemenger introduced a different parameter $\delta_{J}$ to describe axial deformations [7], which is related to $q$ by

$$
q=\frac{2+\delta_{J}}{2-\delta_{J}}, \quad \delta_{J}=2 \frac{q-1}{q+1}
$$

and is only useful for $\delta_{J} \ll 2$. Another definition (see, e.g., [52]) is

$$
q=e^{\delta}
$$

Both definitions Eq. (10) and Eq. (11) have the same lowest-order term, i.e. $q \sim 1+\delta$, in their Taylor expansion for small $\delta$. The diffuse spherical jellium density Eq. (9) can be generalized for spheroidal systems, such that in cylindrical coordinates $(\varrho, z)$

$$
\rho_{J}(\mathbf{r})=\frac{\rho_{0}}{1+\exp \left[\left(\sqrt{z^{2} q^{-4 / 3}+\varrho^{2} q^{2 / 3}}-R_{J}\right) \alpha^{-1}\right]}
$$

The factors containing powers of $q$ ensure that the volume within the underlying sharp spheroid is conserved. The parameter $R_{J}$ in Eq. (12) is fixed by particle number conservation and replaces $R_{I}$ of Eq. (8).

As an alternative, one could define the jellium density in polar coordinates by

$$
\begin{aligned}
& \rho_{J}(r, \theta)=\frac{\rho_{0}}{1+\exp \left[\left(r-R_{J}(\theta)\right) \alpha^{-1}\right]} \\
& R_{J}(\theta)=R_{0}\left[1+b_{0}+\beta_{2} Y_{20}(\theta)+\beta_{4} Y_{40}(\theta)\right]
\end{aligned}
$$

following [53]. Different to Eq. (12), Eq. (13) describes a surface diffusivity which is independent of $\theta$ and $\phi$. Assuming that the ionic mean field is weaker at the top of the spheroidal shape, the zero point motion will have a maximum amplitude in this region, which should be reflected in a smoother radial density profile as chosen in the parametrization Eq. (12).

Since in this work the electronic system is calculated without any constraints except those which arise from the symmetries of the Hamiltonian, it is not surprising that the shape of the electron cloud differs from that of the ionic background. Thus the electronic system cannot be sufficiently well parametrized by just one parameter such as $q$ or $\delta$, but should rather be expanded in a series of even-numbered multipole moments $Q_{0}, Q_{2}, Q_{4}, \ldots$ A multipole decomposition of the electronic density of Na-14 for $0 \leq l \leq 6$ confirms that contributions with $l>4$ are small compared to the leading quadrupole and hexadecupole components (see Fig. 1 below). Thus we can expect that merely quadrupole and hexadecupole contributions are involved in the ground state as long as we restrict ourselves to spheroidal shapes of the jellium density.

The quadrupole and hexadecupole moments of a sharp-edged spheroid can be calculated by formulas derived from Eq. (31) in the appendix:

$$
Q(X)=\frac{2}{5} r_{s}^{2} Z^{5 / 3}\left(q^{4 / 3}-q^{-2 / 3}\right)
$$


Fig. 1 Na-14: Multipole decomposition of the electronic particle density for $l=0,2,4$, 6 in units of [a.u. ${ }^{-3}$ ]. Due to the spheroidal constraint of the jellium, the expansion of the electronic density is well-converged for $l \leq 6$.
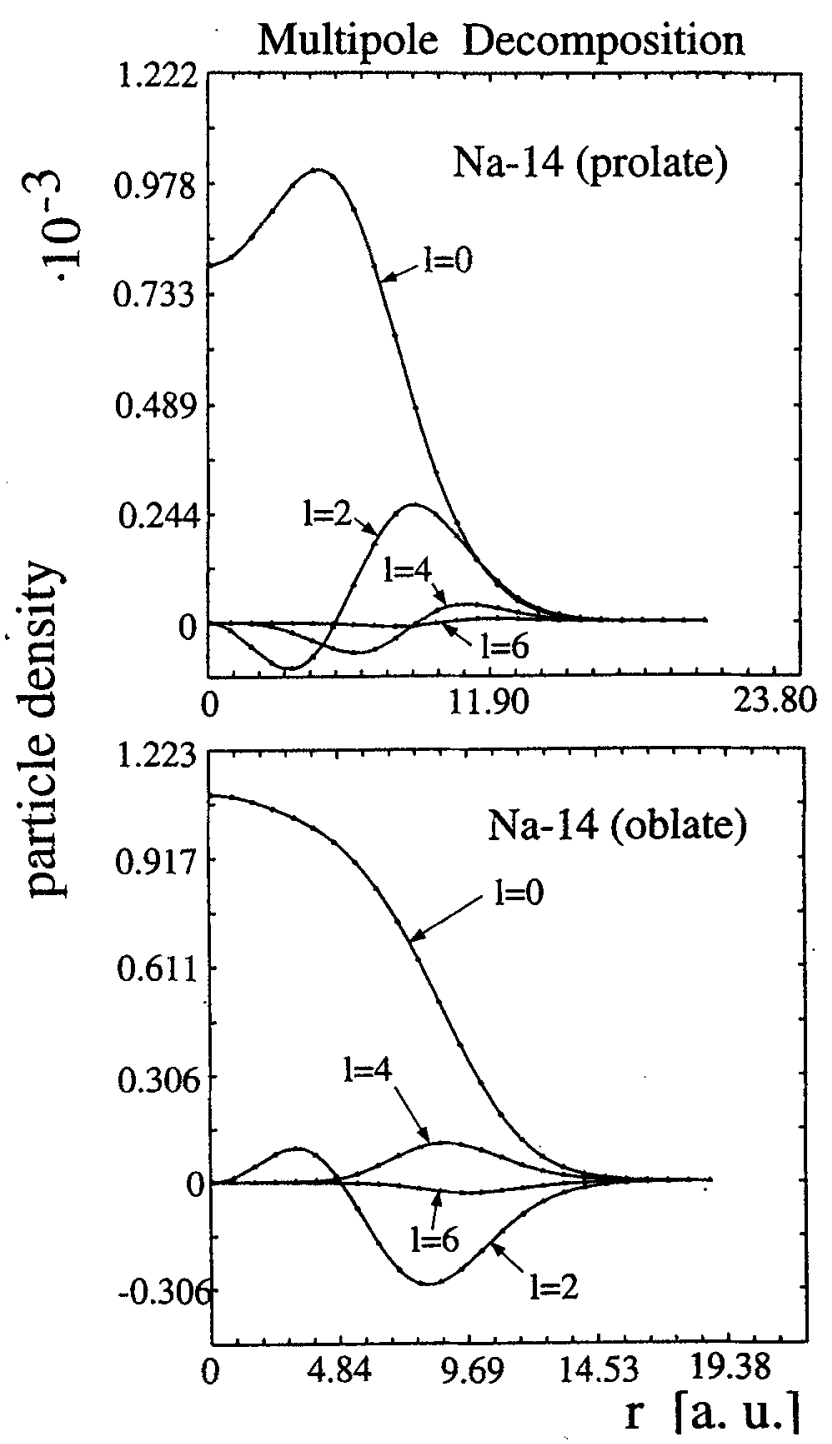

$$
Q_{40}^{(i)}=\frac{9}{70} \sqrt{\pi^{-1}} r_{s}^{4} Z^{7 / 3} q^{-4 / 3}\left(q^{2}-1\right)^{2} .
$$

We also get analytical expressions for the expansion coefficients $\beta_{2}$ and $\beta_{4}$ Eq. (37):

$$
\beta_{2}^{(j)}=\sqrt{\frac{5 \pi}{4}} \frac{q^{2 / 3}}{1-q^{2}} \begin{cases}3-\frac{q^{2}+2}{\sqrt{1-q^{2}}} \operatorname{arccosh}\left(q^{-1}\right) & \text { if } q<1 \\ 3-\frac{q^{2}+2}{\sqrt{q^{2}-1}} \arccos \left(q^{-1}\right) & \text { if } q>1\end{cases}
$$

and 


$$
\beta_{4}^{(j)}=\frac{3}{64} \sqrt{\pi} \frac{q^{2 / 3}}{\left(1-q^{2}\right)^{2}}\left(3\left(3 q^{4}+24 q^{2}+8\right) \mathscr{F}-10\left(11 q^{2}+10\right)\right)
$$

with

$$
\mathscr{F}= \begin{cases}\frac{2}{\sqrt{1-q^{2}}} \operatorname{arccosh}\left(q^{-1}\right) & q<1 \\ \frac{2}{\sqrt{q^{2}-1}} \arccos \left(q^{-1}\right) & q>1 .\end{cases}
$$

Due to the fact that we use the diffuse density distribution Eq. (12), it is impossible to give simple relations for $Q_{e=2,4}^{J}$. In the appendix we show that, given particle number $Z$ and diffusivity $\alpha$, the quadrupole moment $Q_{2}^{(J)}$ of the diffuse and the rectangular jellium only differs by a constant factor. This factor can be taken from Fig. 2 in the particle range from $Z=10, \ldots, 70$ with $\alpha=0.8,1.0,1.2$ a.u.

\subsection{Solution of the Kohn-Sham equations in a deformed basis}

We solve the Kohn-Sham equations by expansion of the wave functions in a set of basis wave functions

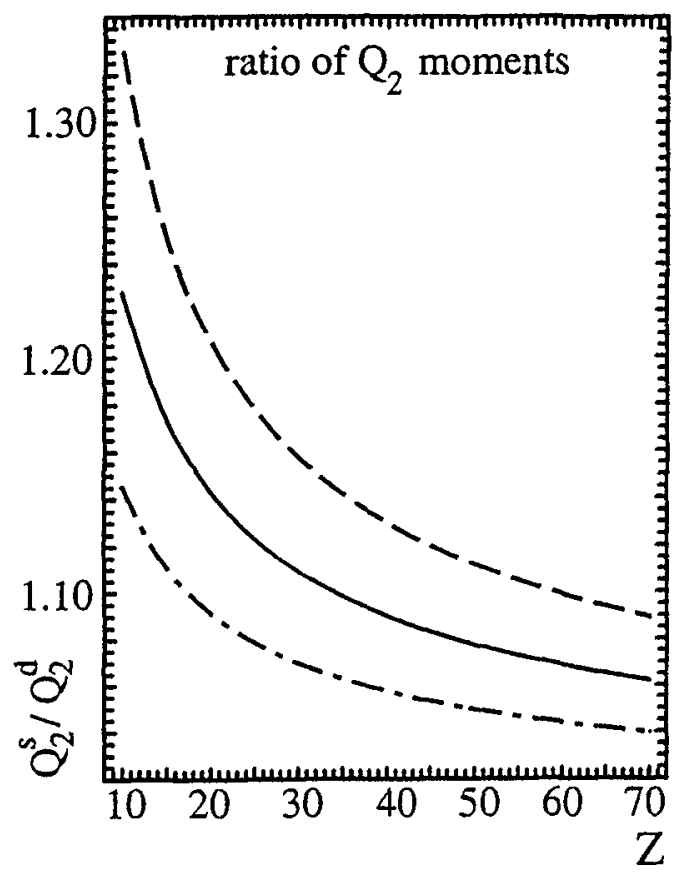

Fig. 2 Ratio of quadrupole moments of a sharp-edged spheroid and a spheroid with a diffuse Fermi-like surface. Solid line: $\alpha=1.0$ a.u. (used in all calculations except otherwise mentioned). Dashed line: $\alpha=1.2$ a.u. Dashed-dotted line: $\alpha=0.8$ a.u. Note that the ratio of the quadrupole moments is independent of the deformation strength $\delta$ for fixed particle number and $\alpha$. 


$$
\phi_{i}(z, \varrho)=\sum_{\alpha} c_{\alpha} \Psi_{\alpha}^{(i)}(z, \varrho)
$$

of an axially deformed harmonic oscillator potential

$$
V(\varrho, z)=\frac{1}{2} m \omega_{\perp}^{2} \varrho^{2}+\frac{1}{2} m \omega_{z}^{2} z^{2}
$$

with the spectrum of eigenvalues

$$
\varepsilon_{n_{Q}, n_{z}}^{|\Lambda|}=\hbar \omega_{\perp}\left(2 n_{Q}+|\Lambda|+1\right)+\hbar \omega_{z}\left(n_{z}+\frac{1}{2}\right) .
$$

with $n_{e}, n_{z}=0,1,2, \ldots$ and $\Lambda=0, \pm 1, \pm 2, \ldots$ Defining the principal quantum number

$$
N_{0}=2 n_{Q}+|\Lambda|+n_{z}
$$

one can rewrite Eq. (22) following the notation of [7]:

$$
\varepsilon \sum_{N_{0}, n_{z}}^{\wedge \mid}=\hbar \omega_{\perp}\left(N_{0}-n_{z}+1\right)+\hbar \omega_{z}\left(n_{z}+\frac{1}{2}\right) .
$$

With $|\Lambda|=0$, one has a maximum degeneracy of 2 , whereas $|\Lambda|>0$ leads to a degeneracy of 4 (including spin degrees of freedom). The standard procedure to optimize the basis system is to increase the number of basis functions in the expansion Eq. (20) until the difference in the total energy becomes negligible. For a detailed discussion of the optimization procedure we refer to the appendix.

Since the calculation of the matrix elements in coordinate space is a very time-consuming procedure, one has to use symmetry properties to spare calculation time. According to [54] and [24], we stay within left/right symmetry, axiality and time reversal invariance.

In case of axial symmetry, the $\phi$-dependent part of the Schrödinger equation can be separated because the z-projection $\Lambda$ of the total angular momentum onto the $z$-axis remains a good quantum number. The total Hamiltonian is block diagonal, each block being indexed by one value of the quantum number $\Lambda$.

As a further consequence of axiality, one has time reversal invariance only for $j_{z}$. The operator $j_{z}$ acting on the $\phi$-dependent part of the one-particle wave function with quantum number $\Lambda_{i}$ gives the same result as the time-reversed operator $-j_{z}$ to the state $-\Lambda_{i}$ [54]. Therefore the corresponding eigenvalues to the quantum numbers $\Lambda$ and $-\Lambda$ for pairs of particles with antiparallel spin are degenerate. Consequently, it is only necessary to sum over positive values of $\Lambda$ in Eq. (20), multiplying the result by 2 [54]. One has to keep in mind that this argument remains exact only for spinsaturated systems.

If we demand left/right symmetry for the electronic density, the corresponding wave functions have either positive or negative parity. Consequently, each block of the total Hamiltonian separates into two subblocks, sandwiched between wave functions with either even or odd parity. 


\section{Static ground-state properties}

We have performed calculations for neutral sodium clusters $(Z=N)$ in the range $8 \leq N \leq 40$. Fig. 3 shows the total energy per electron of each cluster (with even $Z$ ), plotted versus the number $Z$ of valence electrons. The ground-state deformations are obtained with our present diffuse jellium model using different restrictions on their shape. For reference, the short-dashed line indicates the results with imposed spherical shape. It exhibits the familiar minima at the "spherically-magic" numbers 8,20 and 40 . The solid line gives the results of the present calculations with spheroidal shapes, whereas the dashed-dotted line gives results obtained in the triaxial quadrupole-deformed code [22] in the range $8<Z<20$. The long-dashed line finally shows the results obtained in semiclassical density variational calculations [55] with spherical symmetry.

Clearly, the shell effects become less pronounced if deformed shapes are allowed in the model. The largest gain in binding energy is obtained in going from spherical to spheroidal shapes in the regions between the spherically magic clusters, as already observed by Penzar and Ekardt [21, 24]. Only very little extra energy is gained in the triaxial configurations of the clusters with $Z=12$ and 16 . The curve corresponding to the lowest symmetry is rather closely fitted on the average by the semiclassical result which, by construction, does not include any shell effects.

The dips at $Z=14$ and 26 can be taken as weak "deformed-magic" shell closures, exhibiting a locally increased binding energy of deformed clusters. Similar deformedmagic shells have recently been found for large clusters with $N$ up to $\sim 800$ in a spheroidally deformed Nilsson model [52]. Most of the corresponding dips in the total energy have actually been found to agree with similar structures in experimental mass yields [56] after a careful re-examination of the data [52].

Total energy/particle

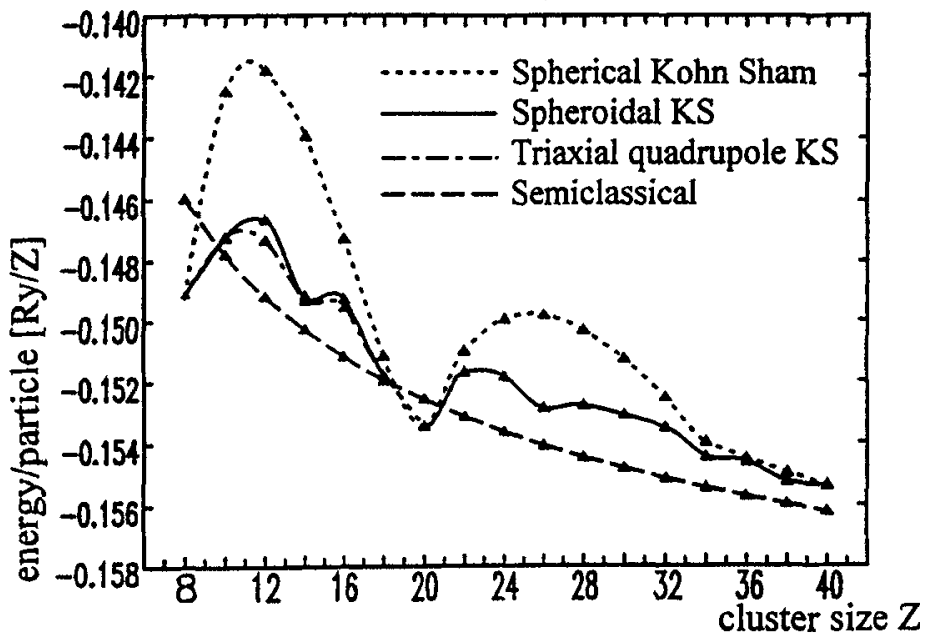

Fig. 3 Total energy per particle obtained from four different calculations with the same diffuse jellium model. Spherical KS: Kohn-Sham results with imposed spherical symmetry. Spheroidal KS: Kohn-Sham results of the present spheroidal jellium model. Triaxial quadrupole KS: Kohn-Sham results for triaxially quadrupole-deformed shapes after [22]. Semiclassical: Semiclassical density variational results using the extended Thomas-Fermi model [55]. Triangles indicate the calculated clusters; the various lines are interpolations. 

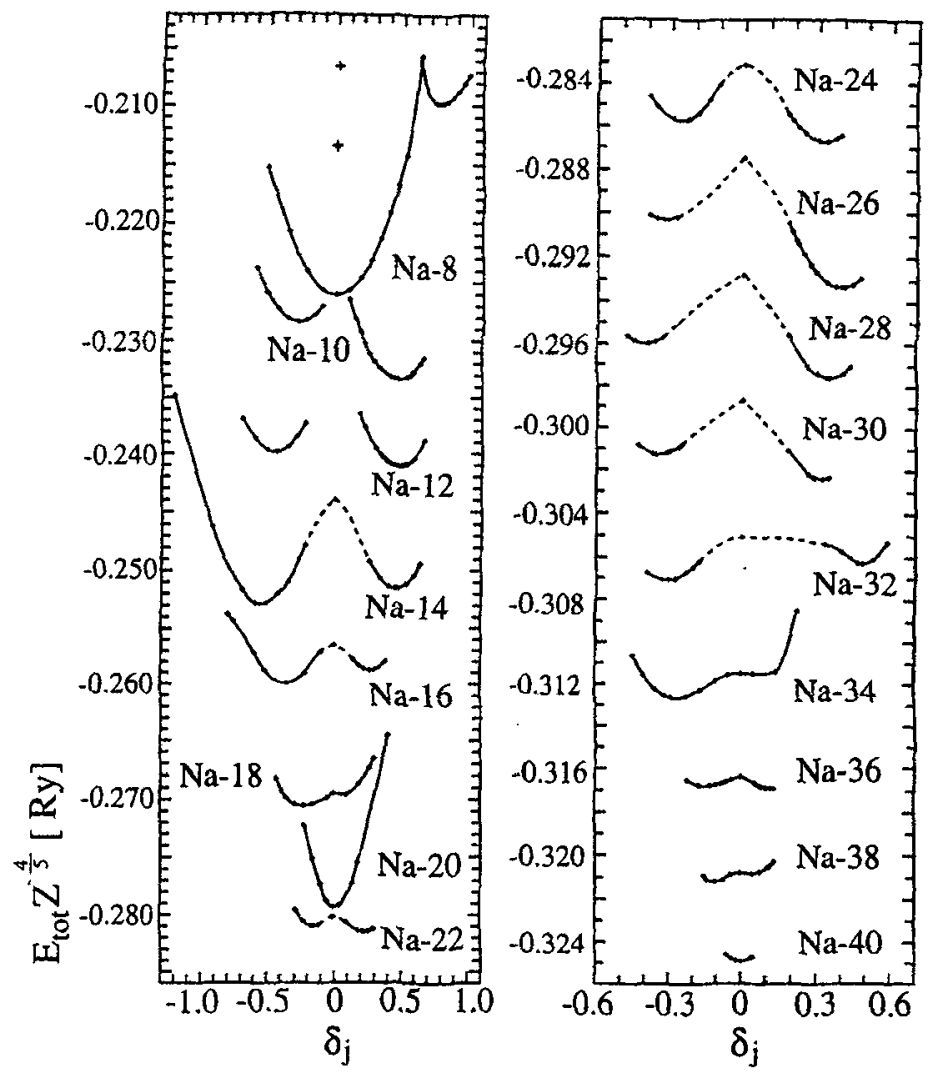

Fig. 4 Deformation dependence of $E_{\text {tot }}$. All curves are scaled by a factor $\left[2^{-4 / 5}\right]$ to fit into one plot. The deformation parameter is defined as $\delta_{j}=\log q$. The upper isolated crosses on the left part are taken from analogous spherical Kohn-Sham calculations for $\mathrm{Na}-10$ and $\mathrm{Na}-12$. Dashed lines lead to the corresponding spherical Kohn-Sham values. As the values obtained from spherical calculations fit very well into the plot, the results can be regarded as well-converged.

\subsection{Potential energy curves}

In Fig. 4 the total energy $E_{t o t} / Z^{4 / 5}$ is plotted versus the deformation parameter $\delta$ defined in Eq. (11). The dashed lines interpolate to the energies which were obtained with the spherical code of Ref. [13] and represented by the points at $\delta=0$. (The isolated spherical points in the upper left correspond to $\mathrm{Na}-10$ and $\mathrm{Na}-12$.)

Our calculations have been performed with a total number of main shells $N_{0}=12$ for Na-8 to Na-20 on a $32 \times 32$ mesh, $N_{0}=14$ for Na-22 to Na-34 with $64 \times 64$ mesh points, and up to $\mathrm{Na}-40$ for $N_{0}=16$ on the same mesh.

$\mathrm{Na}-8, \mathrm{Na}-20$ and $\mathrm{Na}-40$ each reveal spherical shell closures, in accordance with [17], [24], [52] and [7]. The electronic shell closure is confirmed by MD calculations [17] for $\mathrm{Na}-8$ and $\mathrm{Na}-20$. However, the ionic structure was found asymmetrical, which suggests that the electronic behaviour is not very sensitive to the position of the ions. For Na-8, a prolate isomer can be seen in Fig. 4. Oblate-to-prolate transitions occur between $\mathrm{Na}-12 / \mathrm{Na}-14$ and $\mathrm{Na}-30 / \mathrm{Na}-32$, respectively. The small difference in energy, however, makes it difficult to determine the exact particle number at which the transitions exact- 
ly take place, since the energy difference of prolate and oblate isomers slightly depends on assumptions concerning the details of the potential, e.g. the choice of the diffusivity in the definition of $V_{J}(z, \varrho)$, which implies an angular dependence $d(\Omega)$ in our calculations different to, e.g., the definition in [22]. Nevertheless, comparing the results with those of [24] and [52], prolate-to-oblate transitions are kept at about the same particle number in all three models. The absolute value of the deformation strength $\delta$ increases from $\mathrm{Na}-10$ to $\mathrm{Na}-14$. When the d-level is about half-filled at $Z=14$, the deformation is maximum. After $\mathrm{Na}-14$, the distance to the next spherical configuration is decreasing and the minima are moving closer to the spherical point at $\delta=0$. The scheme is repeated from Na-22 until the second deformed shell closure appears at Na-26 (also see Fig. 3). Continuing with $\mathrm{Na}-26$, there is also a tendency to decrease the deformation strength until the second prolate-to-oblate transition takes place at $\mathrm{Na}-30 / \mathrm{Na}-32$, when the electronic configuration changes to the oblate side.

$\mathrm{Na}-36$ slightly favours a prolate deformation with two oblate neighbours. From the near-degeneracy we conclude that Na-36 is triaxially deformed. The situation is similar to the case of $\mathrm{Na}-16$, which also immediately follows a spheroidal shell closure. Na-16 has already been calculated by [57] and found triaxial, having a negative quadrupole moment.

It is interesting to note that for $\mathrm{Na}-18 \mathrm{~s}-\mathrm{d}$ hybridization was also seen in MD calculations [17]. As already illustrated by the corresponding level scheme, orbital mixing (s- $d$ hybridization) is responsible for the non-sphericity of $\mathrm{Na}-18$, and, of course, for $\mathrm{Na}-34$ (f-p hybridization).

\subsection{Role of higher multipolarities}

We have constructed the jellium density as a spheroid with a diffuse surface. Hereby, its multipole moments $Q_{2}^{(j)}$ and $Q_{4}^{(j)}$ have a pre-fixed relation. On the other hand, we did not impose any constraints upon the electronic density except those which are built into the basis (i.e., left-right, time reversal and axial symmetry). Thus, the electrons are free to choose the even multipolarity moments $Q_{4}^{(e)}, Q_{6}^{(e)}$, etc. which minimize their energy. The influence of higher multipolarities on the total energy can be studied qualitatively by comparing the different multipolarities of the jellium and electronic density.

The effect of the multipole moments $Q 20, Q\})$ and $Q 40$ on the total energy was investigated in Ref. [58] in a phenomenological model where a Woods-Saxon potential, fitted to selfconsistent spherical Kohn-Sham potentials, was adapted to deformed shapes. In this model, octupole deformations become prominent in the vicinity of spherical shell closures, e.g. for $Z>40$. From these results one could argue that, formally speaking, the total energetic minimum should be determined by variation of energy within a multidimensional deformation space. Solving this problem selfconsistently, however, would require a tremendous numerical effort. Nevertheless, the deviation of some electronic multipolarities $Q_{2}^{(e)}, Q_{4}^{(e)}$ from those of the jellium background allows one to judge whether the true deformed minimum has been obtained or not - if the electronic system does, not prefer further asymmetries.

Fig. 3 shows the dependence of the total energy per particle on the symmetries involved. Obviously, the spherical Kohn-Sham calculation [50] is worst when the clusters have triaxial shape, such as, e.g., $\mathrm{Na}-12$ and $\mathrm{Na}-16$ which have been found to be triaxial (in [22] and [57]). On the other hand, triaxial corrections to the total energy appear 
to be small in magnitude compared with the energy gain obtained with spheroidal deformations, as long as only quadrupole deformations $Q_{2 m}(m=0, \pm 2)$ with conserved left/right symmetry are concerned. The small cusp between Na-34 and Na-38 gives an idea of the magnitude of the triaxial corrections for larger clusters.

\subsection{1 $Q_{2}$ Systematics for $8 \leq Z \leq 40$}

Table 1 shows the systematics of equilibrium deformations $\delta$ of axial clusters in the region $8 \leq Z \leq 40$, obtained in the present work (column "diffuse jell.") and in calculations with related models (columns b, $c, d$ ). Column a) indicates the shapes deduced from a recent analysis of collective resonances observed in photoabsorption experiments [59].

Different jellium shapes can lead to different quadrupole moments for equally chosen $\delta$. In particular, the quadrupole moment of a volume-conserved spheroid with a diffuse surface is smaller than that of the corresponding sharp-edged spheroid. This should be taken into consideration when comparing the values of $\delta$ from different models. Therefore their precise values should not be taken too literally. The general systematics, however, are found to agree well between the different models. Also, comparing to the analysis of Ref. [59], we see that the predicted shape transitions in Tab. 1 are in good accordance with the experimental ones. It is quite astonishing that already the simple ellipsoidal model [23] seems to predict correctly the triaxiality of Na-36.

Table 1 Systematics of equilibrium deformations $\delta$ and shape transitions (Values of $\delta$ in brackets refer to the energetically higher isomer.)

\begin{tabular}{rllllll}
\hline$Z$ & a) & diffuse jell. & shape & b) & c) & d) \\
\hline 8 & - & 0 & $\mathrm{~s}$ & 0 & 0 & - \\
10 & - & $(-0.29), 0.46$ & $\mathrm{p}$ & 0.44 & 0.48 & $(-0.30), 0.35$ \\
12 & - & $(-0.42), 0.45$ & $\mathrm{p} / \mathrm{d}$ & $\mathrm{t}$ & - & $-0.45,(0.40)$ \\
14 & - & $-0.54,(0.44)$ & 0 & -0.50 & -0.56 & $-0.30,(0.35)$ \\
16 & 0 & $-0.35,(0.26)$ & $\mathrm{o} / \mathrm{d}$ & $\mathrm{t}$ & - & $-0.35,(0.65)$ \\
18 & $\mathrm{o}$ & -0.22 & 0 & -0.24 & -0.17 & $-0.20,(0.50)$ \\
20 & $\mathrm{~s}$ & 0 & $\mathrm{~s}$ & 0 & 0 & 0 \\
22 & $\mathrm{p}$ & $(-0.14), 0.23$ & $\mathrm{p}$ & 0.25 & 0.22 & $(-0.15), 0.20$ \\
24 & $\mathrm{p}$ & $(-0.23), 0.32$ & $\mathrm{p}$ & $\mathrm{t}$ & - & $(-0.25), 0.30$ \\
26 & $\mathrm{p}$ & $(-0.31), 0.39$ & $\mathrm{p}$ & 0.39 & 0.37 & $(-0.60), 0.35$ \\
28 & $\mathrm{c}$ & $(-0.35), 0.35$ & $\mathrm{p}$ & $\mathrm{t}$ & - & $(-0.40), 0.35$ \\
30 & $\mathrm{c}$ & $(-0.35), 0.32$ & $\mathrm{p}$ & $\mathrm{t}$ & - & $(-0.35), 0.30$ \\
32 & $\mathrm{o}$ & $-0.30,(0.46)$ & $\mathrm{p} / \mathrm{d}$ & $\mathrm{t}$ & - & $-0.30,(0.30)$ \\
34 & $\mathrm{o}$ & $-0.26,(0.05)$ & 0 & -0.31 & -0.18 & $(-0.30), 0.20$ \\
36 & $\mathrm{o}$ & $(-0.15), 0.10$ & $\mathrm{p} / \mathrm{d}$ & $\mathrm{t}$ & - & $(-0.10), 0.10$ \\
38 & $\mathrm{o}$ & $-0.11,(0.05)$ & 0 & -0.13 & -0.09 & $-0.40,(0.60)$ \\
40 & $\mathrm{~s}$ & 0 & $\mathrm{~s}$ & 0 & 0 & 0 \\
\hline
\end{tabular}

a) Experimental shapes obtained from collective resonances [59]

b) Triaxial harmonic oscillator model [23].

c) Selfconsistent spheroidal model [24].

d) Schematic model with potential according to Eq. (9) (unpublished).

$(s=$ spherical, $o=$ oblate, $p=$ prolate, $t=$ triaxial, $d=$ nearly degenerate isomers, $c=$ coexisting shapes). 


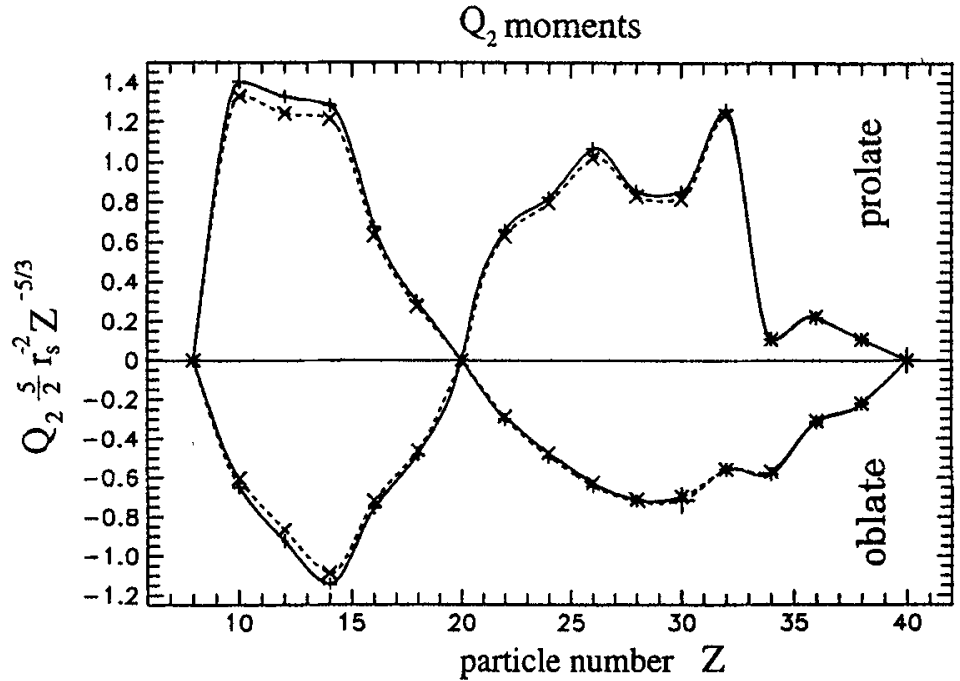

Fig. 5 Quadrupole moments of electrons $Q_{2}^{(e)}$ (crosses, dashed line) and ions $Q_{2}^{(j)}$ (plus-signs, continuous line) (in units of $\frac{2}{5} r_{s}^{2} Z^{5 / 3}$, which is the size-dependent part of the corresponding quadrupole moment of the spheroid Eq. (16)), versus particle number $Z$. Shape transitions take place at $Z=12-14$ (prolate-oblate), $Z=18-20-22$ (oblate-spherical-prolate), and $Z=30-32$ (prolateoblate).

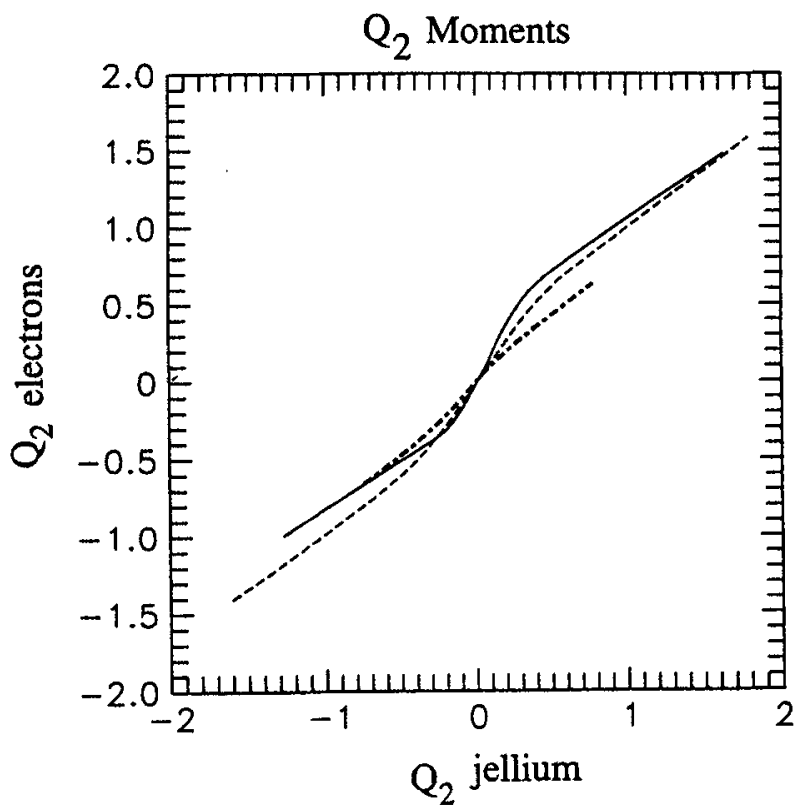

Fig. 6 Quadrupole deformations of the jellium and the electronic system for Na-10, Na-14 and $\mathrm{Na}-18$, plotted in the dimensionless units of Fig. 5. Continuous line: Na-14. Dashed line: Na-10. Dashed-dotted line: Na-18. 


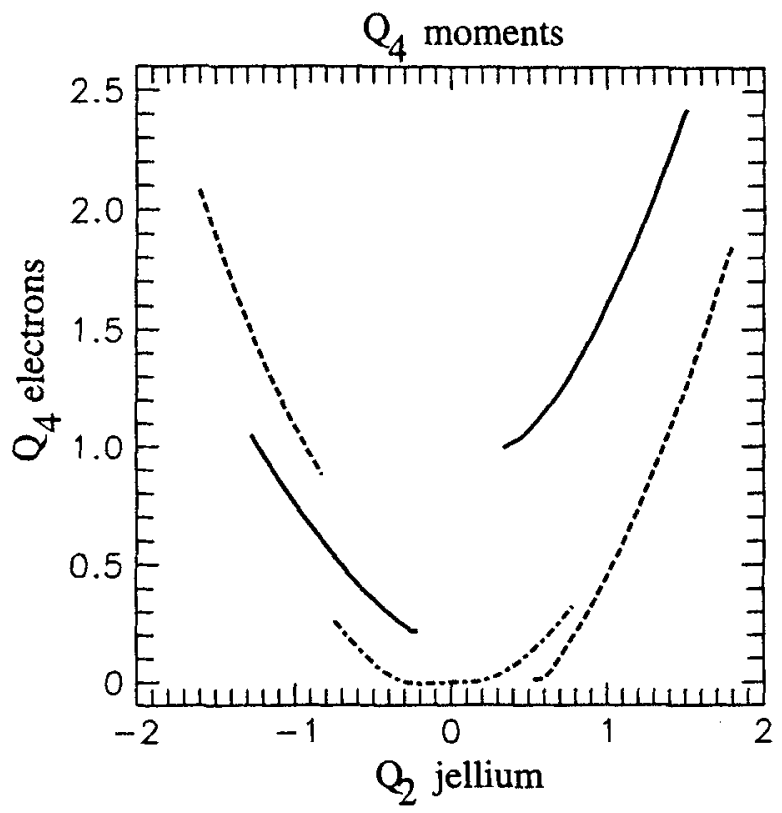

Fig. 7 Electronic hexadecupole moment $Q_{4}^{(e)}$ versus the ionic quadrupole moment $Q(j)$ for $\mathrm{Na}-10$, $\mathrm{Na}-14$ and $\mathrm{Na}-18$ (we refer to the analogous $Q_{4}$ value of the spheroid Eq. (16) for the definition of the dimensionless units [const. $r_{s}^{4} Z^{7 / 3}$ ], with const. $=9 /(70 \sqrt{\pi})$. Continuous line: Na-14. Dashed line: Na-10. Dashed-dotted line: Na-18. Note that Na-14 tries to conserve an intrinsic electronic hexadecupole moment in the ground state.

\subsection{2 $Q_{2}^{(e)}$ versus $Q(j)$}

Fig. 5 shows the electronic and ionic quadrupole moments $Q_{2}^{(e)}$ and $Q_{2}^{(j)}$, respectively, of sodium clusters with $8 \leq Z \leq 40$ at their prolate and oblate (or spherical) equilibrium deformations. The overlap of the two curves indicates that the clusters at the equilibrium deformations have almost equal shapes of electrons and ions, as long as no other ionic multipolarities are considered. For small deviations from the equilibrium deformations, the dependence of $Q_{2}^{(e)}$ on $Q_{2}^{(j)}$ is found to be linear, as shown in Fig. 6 for the three clusters $\mathrm{Na}-10, \mathrm{Na}-14$ and $\mathrm{Na}-18$. For the strongly deformed $\mathrm{Na}-10$, we see that there is an over-proportional increase of $\left|Q_{2}^{(e)}\right|$ in the vicinity of the spherical point: the electronic system tries to conserve the deformed shape as $Q 2(j)$ vanishes.

Although one observes only small differences between $Q_{2}^{(j)}$ and $Q_{2}^{(e)}$, it becomes obvious (see Fig. 7 below) that these differences become stronger when finite hexadecupole moments evolve.

\subsection{3 $Q_{4}^{(e)}$ versus $Q 4^{(j)}$}

For some particular clusters, the hexadecupole degree of freedom has been found to play a major role. In Fig. 7 we present the electronic hexadecupole moment $Q_{4}^{(e)}$ as a function of $Q_{2}^{(j)}$ for the same three clusters as in Fig. 6. The relation is far from being linear here: we learn from Fig. 7 that the prolate isomer of $\mathrm{Na}-10$ tries to conserve a 


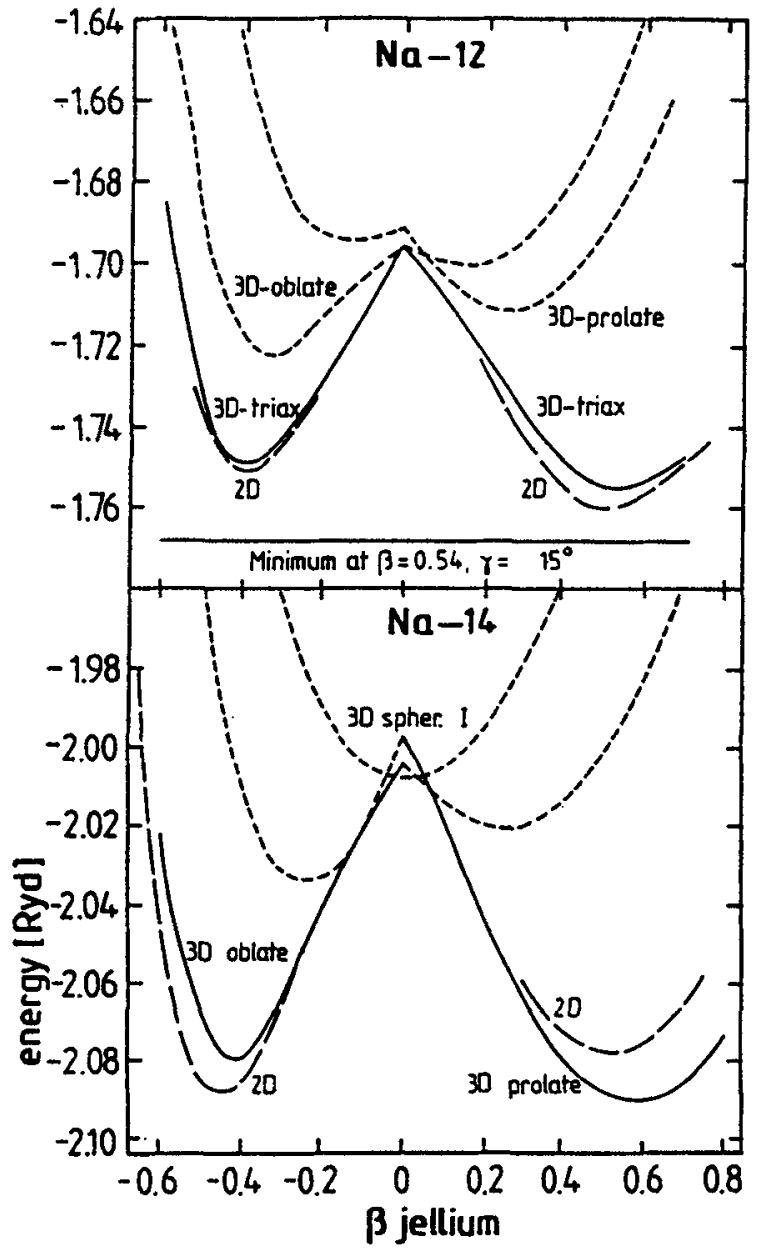

Fig. 8 Total energy of Na-14 and Na-12. Continuous line: triaxial calculation with $\left.Q 2()_{2}\right)$ only (see Ref. [22] for details). Dashed line: axial calculation with $Q X^{\prime} \neq 0$. Differences in the total energy are related to the influence of an electronic hexadecupole moment.

finite $Q_{4}^{(j)}$ moment, whereas Na-14 conserves a $Q_{4}^{(e)}$ moment on the oblate side. ${ }^{3}$ We can also see that for the deformed minima of Na-10 and $\mathrm{Na}-14$, where the differences between the quadrupole moments of electrons and ions are largest (see Fig. 5), the electrons have developed a considerable nonzero hexadecupole moment.

The role of the electronic hexadecupole moment of $\mathrm{Na}-14$ also explains the discrepancy of some earlier results obtained by Lauritsch et al. [22] in two slightly different models. In Fig. 8, we show their deformation energies for $\mathrm{Na}-12$ and $\mathrm{Na}-14$, plotted against the jellium deformation parameter $\beta_{j}$. The curves labeled 2D were anticipated from our present calculations with the two-dimensional axial code, whereas the three-dimensional ( $3 \mathrm{D})$ results were obtained in triaxial calculations for quadrupole shapes in the Hill-Wheeler parametrization $(\beta, \gamma)$. In these $3 \mathrm{D}$ calculations, which confirm that $\mathrm{Na}-14$ stays axial at both minima (in contrast to $\mathrm{Na}-12$, whose ground state has a triaxial shape), the jellium density profile was defined similarly to Eq. (12) with

${ }^{3}$ For the definition of $\beta_{2}^{\left.()^{\prime}\right)}$, see Eq. (37). 


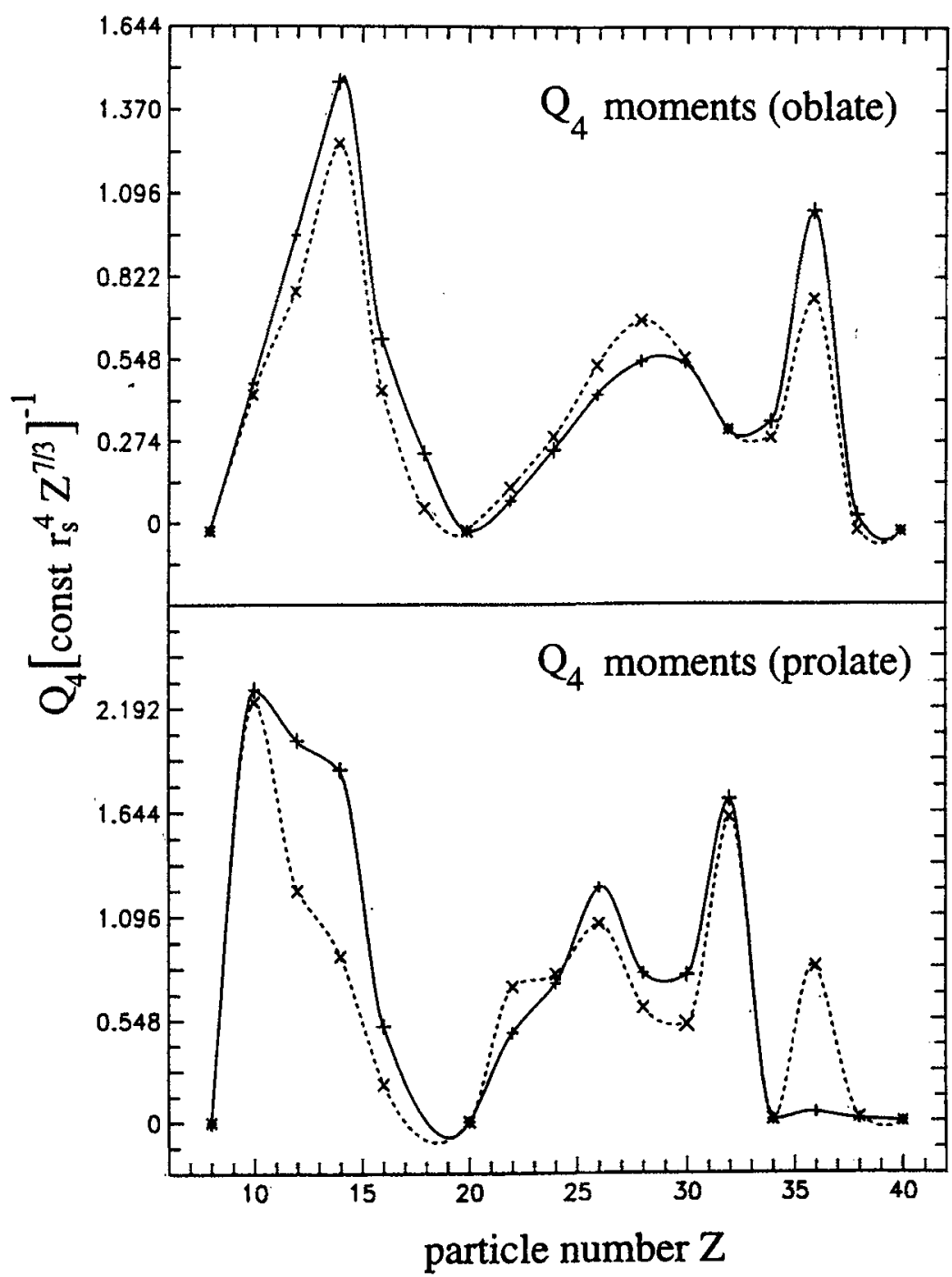

Fig. 9 Hexadecupole moments for $8 \leq Z \leq 40$ (in dimensionless units according to Fig. 7). Dashed lines, crosses: $Q_{4}^{(e)}$. Continuous lines, plus-signs: $Q()^{\prime)}$. Different hexadecupole moments reveal a strong $Q_{4}$-dependence of the ground state.

the same $\alpha=1.0$ a.u. However, the expansion coefficients in Eq. (14) were adapted such that $Q()^{(j)}=0$. As a consequence, the missing $Q_{4}^{(j)}$ moment for the oblate isomer of Na-14 in the triaxial calculation implies a bad overlap of the electronic and the jellium density. The prolate isomer, on the other hand, prefers a quadrupole deformation with a small $Q_{4}^{(e)}$ (see Fig. 7), which worsens the overlap in the 2D result because of the nonvanishing $Q 4$ (j) of the parametrization Eq. (12).

The systematics of hexadecupole moments are shown in Fig. 9. Electronic and ionic hexadecupole moments are substantially different at the particle numbers $Z=12,14$, $22,26,28,30$ and 36 . The strongly differing hexadecupole moments of $\mathrm{Na}-36$ for both isomers suggest that this cluster has a triaxial shape. 


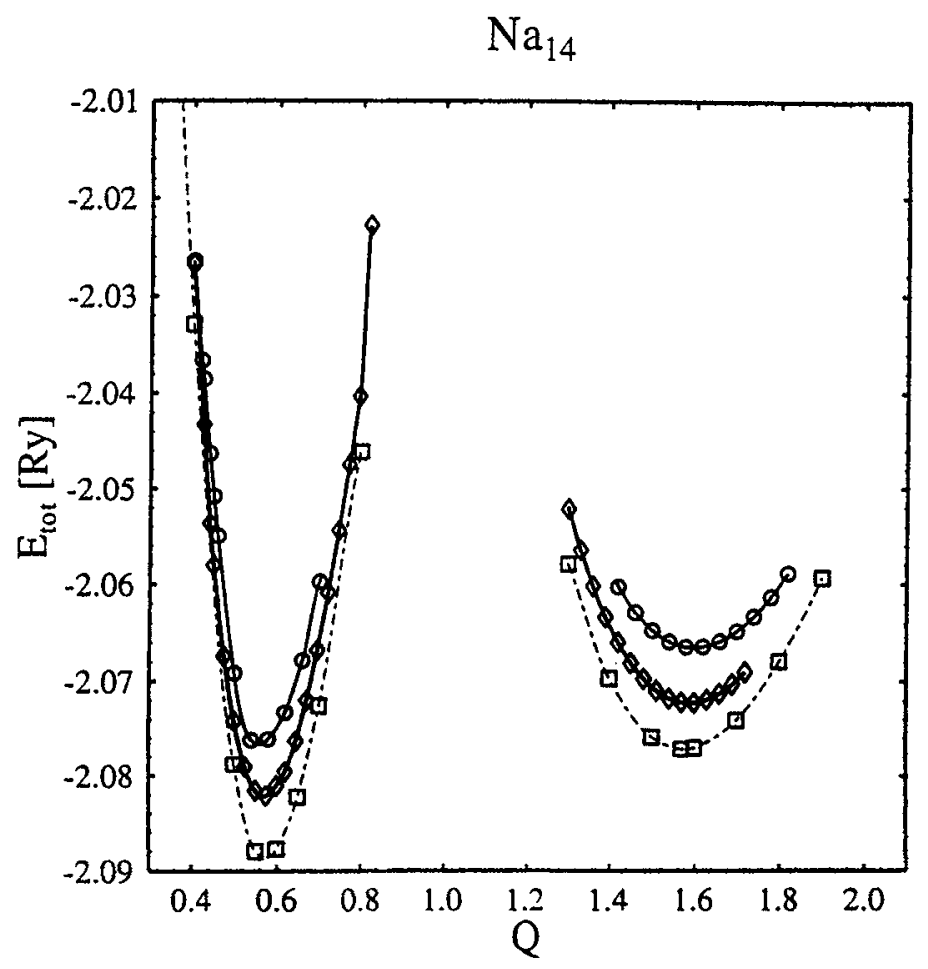

Fig. 10 Na-14: PES curves for three different values of $\alpha$. Thin continuous line, circles: $\alpha=0.70$ a.u. Thick continuous line, rhombi: $\alpha=0.80$ a.u. Dashed-dotted line, squares: $\alpha=1.00$ a.u. Absolute values of $q$ are stable within parts of percentages. Vertical positions of the total energy are only shifted by varying $\alpha$.

We conclude that, as a consequence of these variations in the hexadecupole moments, an explicit variation of the shape of the jellium density in the two-dimensional deformation space $\left(\beta_{2}, \beta_{4}\right)$ of the expansion Eq. (13) should be performed in order to improve the shape systematics. Work along these lines is in progress [48].

\subsection{Influence of surface diffusivity $\alpha$}

In order to investigate to which extent the surface diffusivity $\alpha$ of the jellium density affects the equilibrium deformations and their relative energies, we have calculated some PES curves for various values of $\alpha$. In Fig. 10 we present the results for the welldeformed cluster $\mathrm{Na}-14$ for the values $\alpha=0.60,0.80$ and 1.0 a.u. We observe that the only effect of this variation is a constant shift in total energy, whereas the deformations of the oblate and prolate isomers are unaffected within the numerical precision of the results. It situation was confirmed in other cases with either well-developed static deformations or a pronounced spherically-magic configuration. It means that whenever the electronic single-particle level density has a pronounced minimum at the Fermi energy, leading to a local minimum of the total energy and thus an enhanced stability of the cluster, the solutions are also stable with respect to small changes of the ionic potential such as those caused by the variation of $\alpha$. 


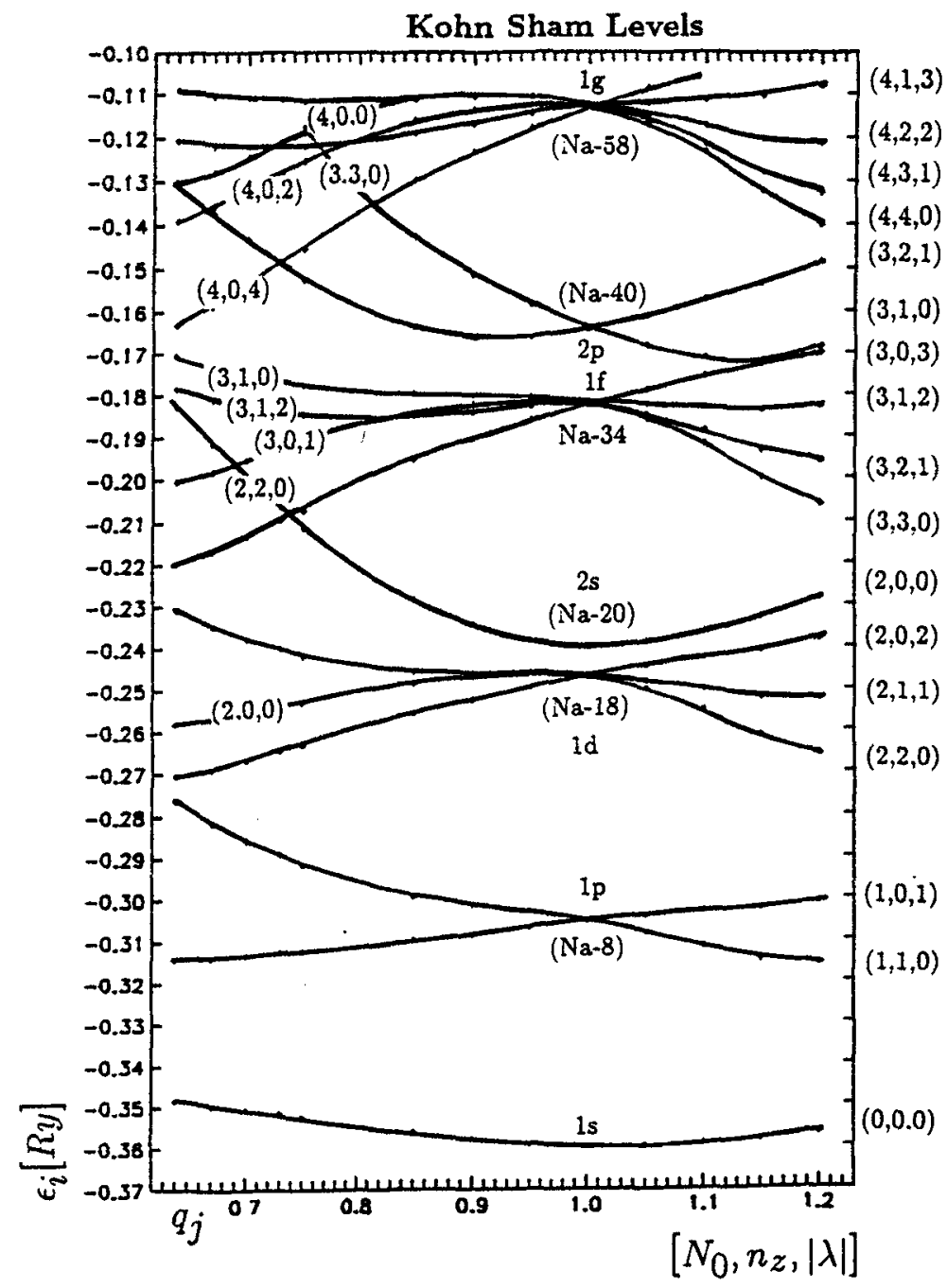

Fig. 11 Deformation dependence of selfconsistent Kohn-Sham levels for Na-34. The 1f-shell is closed. Note that $1 d / 1 \mathrm{~s}$ and $1 \mathrm{f} / 2 \mathrm{p}$ levels are close enough to mix. Levels are classified following the convention of Clemenger using Nilsson's asymptotic quantum numbers.

In intermediate situations, e.g. for Na-34 where there is a strong interaction between the highest occupied (HOMO) and the lowest unoccupied (LUMO) level, the variation of $\alpha$ can have larger effects, as will be discussed in the next paragraph.

\subsection{Kohn-Sham levels}

The influence of deformation on the selfconsistent single-particle levels is illustrated in Fig. 11. The steepness of a considered level $\varepsilon_{\alpha}$ can be taken ${ }^{4}$ as a measure of the quadrupole expectation value of the corresponding single-particle wavefunction [60]:

\footnotetext{
${ }^{4}$ In first order of the deformation $\delta$.
} 


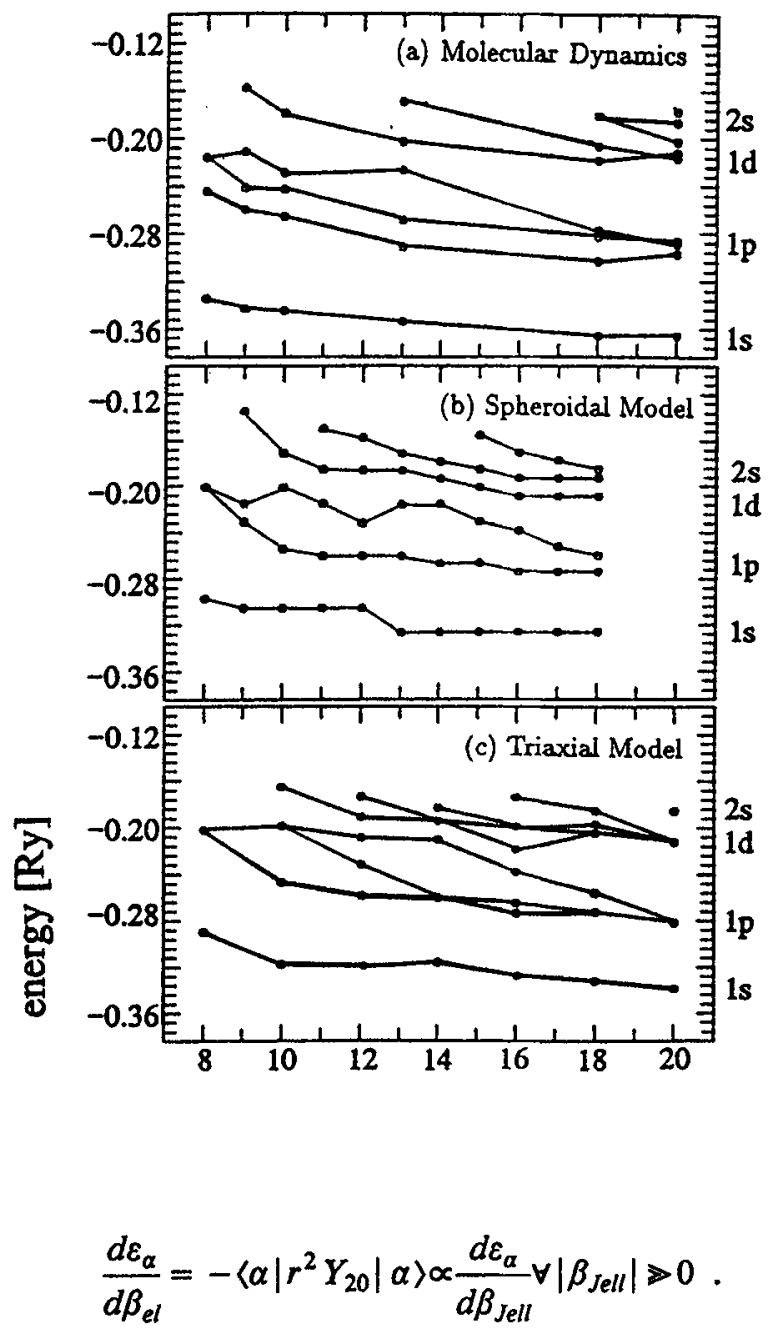

Fig. 12 Kohn-Sham levels for $Z=8$ to 20 . (a) MD calculations by [17], (b) spheroidal model by [24]; (c) diffuse jellium model.

From this we see that prolate deformations give a positive quadrupole moment, whereas oblate deformations are connected with a negative value of $Q_{2}$. Levels which are going down with deformation (considered from oblate to prolate shapes) have a positive (prolate-like) $Q_{2}$ value, uprising levels have a negative (oblate-like) $Q_{2}$ value.

It is interesting to compare the selfconsistent Kohn-Sham levels with the corresponding level schemes which have been derived from modified Nilsson Hamiltonians.

Clemenger [7] proposed a simplified Nilsson-model for alkali clusters without spinorbit coupling. Reimann et al. [52] fitted the Nilsson Hamiltonian to the selfconsistent spherical spectrum, thus keeping some selfconsistency to the results. Although the schematic results are in quite good qualitative agreement, they certainly cannot reproduce the effects of selfconsistency, e.g. the fact that $\partial^{2} \varepsilon_{\alpha} / \partial \beta^{2}$ changes its sign for some levels in the selfconsistent calculation.

The deformation-dependent interaction of the different levels with the jellium can be illustrated, e.g., with the behaviour of the $1 \mathrm{~d}$-levels.

- Levels with small angular quantum numbers $|\Lambda|$ are closer to the z-axis [60]. These levels are favouring the prolate deformation of the jellium, since they have a posi- 
tive $Q_{2}$ moment. On the other hand, levels with high angular momentum prefer oblate shapes, and they are shifted upwards with increasing prolate deformation.

- Levels with quantum numbers $(2,0,0),(2,2,0),(2,0,2)$ and $(2,1,1)$ emerge from the spherical $1 \mathrm{~d}$-level which is five-fold degenerate (without spin). In the case of $\mathrm{Na}-18$, when each $1 \mathrm{~d}$-level is populated, this degeneracy is split into three branches, with doubly degenerate levels for $|\Lambda|>0$.

- The $(2,0,0)$ level interacts with the level from the $2 \mathrm{~s}$ level which has the same symmetry. The interaction shifts this level downwards, being responsible for the nonsphericity of $\mathrm{Na}-18$.

The same situation appears in the $1 \mathrm{f} / 2 \mathrm{p}$ region around Na-34 which has a closed f-shell. The deformed levels $(3,2,1)$ and $(3,1,0)$ interact with levels of the same symmetry in the 2p-shell. This hybridization leads to a static oblate deformation of $\mathrm{Na}-34$, in spite of its filled $f$-shell. The effect becomes even more pronounced in the diffuse jellium model, because the diffuseness smoothes the potential, shifting levels with higher $l_{z}$ upwards and levels with lower $l_{z}$ downwards. As a result, the interaction between these levels increases, and the resulting deformation is also increased by an amount that depends on the value of the diffuseness parameter $\alpha$.

Note that $\mathrm{Na}-34$ is a notorious case in the standard spherical jellium model with sharp edge: it has a stronger "magic" single-particle (HOMO-LUMO) gap than Na-40, contrary to what is known from the experimental mass abundances (see also Refs. [5, 6]). The introduction of a smooth jellium surface, shifting the $1 \mathrm{f}$ and $2 \mathrm{p}$ levels closer together, improves this situation, as was observed already by Rubio et al. [49], making $\mathrm{Na}-34$ less magic and $\mathrm{Na}-40$ more magic.

The Kohn-Sham spectrum of sodium clusters with particle number $8,10,11,13,18$ and 20 obtained by MD calculations of [17] has been compared with the spectrum of the sharp-edged jellium model [24] in [17]. Comparing our results in Fig. 12 with triaxial results calculated by Lauritsch et al. [22] for $\mathrm{Na}-12$ and $\mathrm{Na}-14$, we find an astonishing agreement with the results of the MD calculations: $1 \mathrm{~s}, 1 \mathrm{p}$ and $1 \mathrm{~d}$ levels are almost equally aligned with respect to each other. $1 \mathrm{~d}$ and $1 \mathrm{p}$ levels exhibit a neardegeneracy at the spherical shell closure at $Z=20$, whereas the $1 \mathrm{p}$ level is slightly splitted at $Z=8$ in the MD calculation. ${ }^{5}$ Extrapolating the data of $\mathrm{Na}-13$ of [17] to the first axial subshell closure at $Z=14$, one observes that the $1 \mathrm{p}$ and $1 \mathrm{~d}$ levels are in good quantitative agreement with the MD results.

At $Z=14,1 \mathrm{p}$ levels show a significant level-bunching in the immediate vicinity of a deformed subshell closure. In this model, where left/right symmetry forbids nonvanishing matrix elements between states of different parity, the interaction of these levels is excluded (Fig. 11).

However, a small gap between $1 \mathrm{~d}$ and $1 \mathrm{p}$ levels can lead to a mixing of these levels, if one allows for a static octupole plus quadrupole deformation, which can occur if one introduces a $\left(Q_{3}+Q_{2}\right)$-adapted jellium density. It has been shown by Hamamoto et al. [61] that octupole instabilities in typically quadrupole-deformed regions are a rather general feature of finite fermion systems. It leads to shell effects of comparable importance to those induced by nonaxial quadrupole deformations. Further investigations which break left/right symmetries are the subject of current work.

\footnotetext{
${ }^{5}$ One has to keep in mind, however, that this small cluster is almost beyond description in the simple jellium model.
} 


\subsection{Densities and Kohn-Sham potentials}

A crucial question which affects the applicability of the jellium is still at stake: Do the different selfconsistent KS potentials of the jellium model and the KS potentials obtained by MD calculations lead to comparable electronic densities? In Fig. 13 we compare the spherically averaged total KS potentials of Na-10 and Na-20 obtained by Röthlisberger and Andreoni [17] with the results of the diffuse jellium model. In both calculations, exchange and correlation effects have been taken into account by the same functional of [26] in LDA. The mean radial position of the ions is well resolved in MD calculations because of the microscopic treatment of the ionic influence by pseudopotentials. However, the average trend of the potential is fairly well reproduced by the jellium model. For larger clusters like $\mathrm{Na}-20$ (which still is a small cluster in the jellium model) the oscillations of the ions decrease relatively to the depth of the potential.

The corresponding spherically averaged electronic densities of the MD results, obtained at different temperatures, are given on the left-hand side of Fig. 14, whereas the right-hand side exhibits the present jellium model results. Since here the structure of the ions is completely neglected, it is not surprising that the MD densities at the higher temperatures, where the ionic structure is smeared out, resemble the density distributions of the jellium model more than at zero temperature.

\section{Collective excitations}

Photo-absorption cross-sections of charged sodium clusters reveal resonances similar to the giant isovector $E_{1}$ resonances of nuclei $[62,63]$ which are excited by $\gamma$-radiation and consist of collective dipole oscillations of neutrons against protons. In metal clusters, these resonances can be understood as collective dipole oscillations of the electrons with respect to the ions. If the diameter of the cluster is much smaller than the
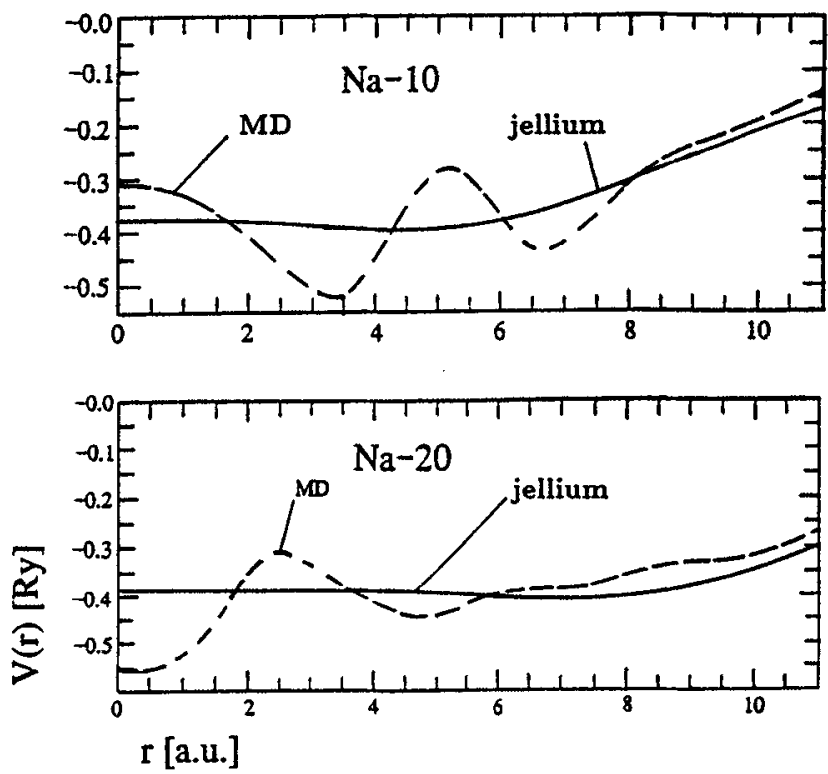

Fig. 13 Spherically averaged MD Kohn-Sham and jellium Kohn-Sham potential. Continuous line: spheroidally deformed jellium model. Dashed line: MD calculation by [17]. The gross structure of the potential is well conserved in the jellium calculation. (Adapted from Ref. [17].) 

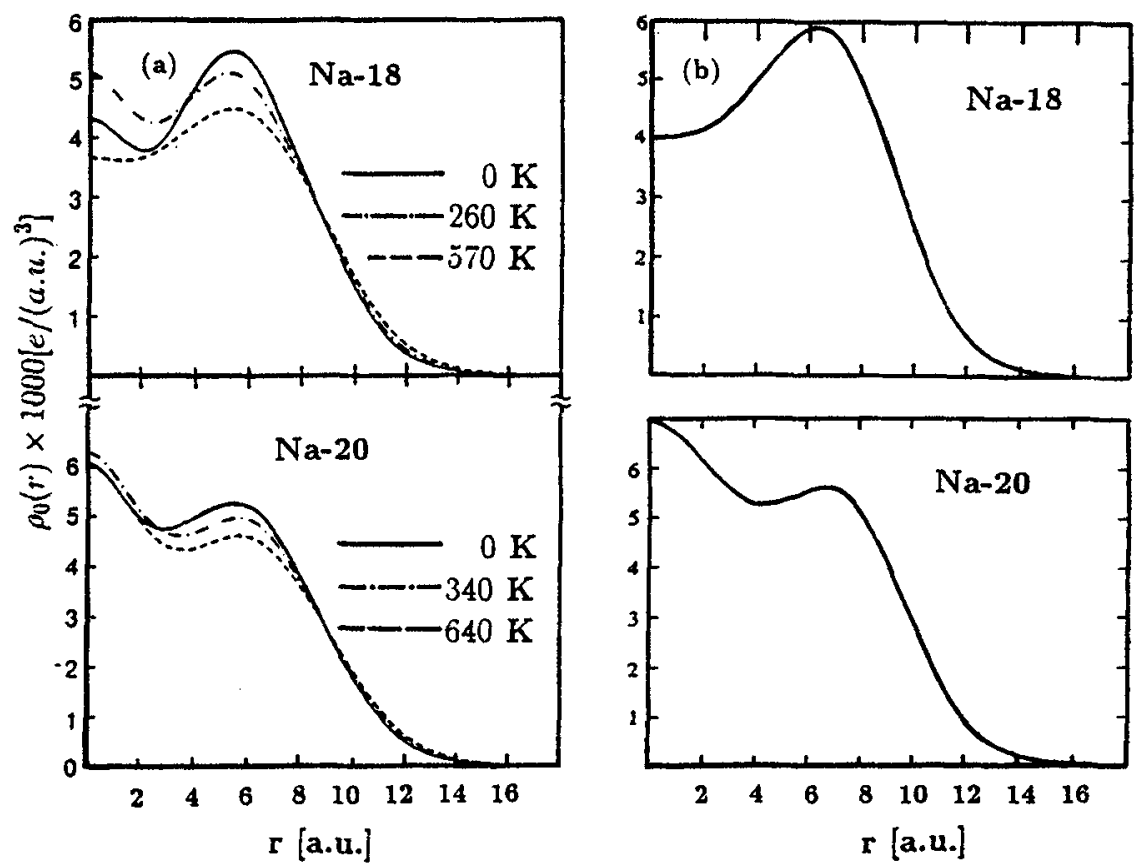

Fig. 14 Spherically averaged densities obtained from (a) MD calculations and (b) the deformed jellium model. The nodal structures of the density distributions of both methods are very similar.

wavelength of the incident light, the electrons are shifted from their equilibrium position along the symmetry axes by the momentum transfer of the dipole component of an incident light beam, and a collective resonance is excited. As the charged ionic background has a much higher mass than the electronic system, it does not respond to the electronic translation within the time scale of the electrons.

To lowest order, the restoring force of such a dipole oscillation is coming from the electron-ion interaction which leads to a purely translational mode with conserved electron density. (This corresponds to the Goldhaber-Teller mode proposed for nuclei [63].) Classically, this mode leads to the so-called "surface plasmon" or "Mie plasmon" of a metal sphere; in fact, the classical theory of its dynamic polarizability predicts a single dipole resonance at the so-called Mie frequency:

$$
\omega_{M i e}=\sqrt{\frac{\hbar^{2} e^{2}}{m r_{s}^{3}}}=\frac{1}{\sqrt{3}} \omega_{b u l k}
$$

The kinetic and Coulomb energies of the electrons (including exchange and correlations) contribute to the dipole restoring forces only if the electronic density is allowed to be locally compressed or decompressed. This leads to "volume plasmon" modes whose macroscopic limit is the bulk plasma frequency $\omega_{\text {bulk }}$. Although an external dipole field does not couple directly to these volume oscillations, their coupling to the surface modes affects the main dipole resonance by giving it an extra red-shift and reducing its collectivity $[6,55]$.

Microscopically, the dipole resonances can be decomposed into $1 \mathrm{p}-1 \mathrm{~h}$ excitations, whose coherent superposition may lead to one excited state which exhausts a large part 
of the dipole sum rule and thus contains most of the collectivity. Indeed, the quantummechanical dipole absorption cross sections of spherical alkali clusters, obtained by jellium calculations in linear response theory (in TDLDA [64] or RPA $[37,65,66]$ ), exhibit a dominant peak which exhausts some $75-90 \%$ of the dipole sum rule, and is red-shifted by $10-20 \%$ with respect to the Mie formula (Eq. (26)). Since the centroid of the RPA strength distribution goes towards the Mie result in the limit of a macroscopic metal sphere [55], the red-shift in finite clusters can be related to the "spill-out" of the electronic wave functions over the jellium edge: screening effects and the absence of positive charge lead to a decrease of the restoring forces for the electrons at the surface of the cluster, shifting the resonance peaks down to lower frequencies.

It is evident that a detailed description of photoabsorption cross sections can only be made in a microscopic theory, such as the TDLDA or RPA methods just mentioned - in particular when a fragmentation of the collective strength into several states can be found, which results from interferences of specific particle-hole states with the collective "Mie plasmon". However, the gross structure of the dipole resonances, in particular their size and shape dependence, is well described by simple sum rule techniques (see, e.g., Ref. [6]).

A sum rule is defined as a sum of transition probabilities from the ground state $|0\rangle$ to the excited states $|n\rangle$, weighted with powers of the transition energy $\hbar \omega_{n}=E_{n}-E_{0}$ :

$$
m_{k}(Q)=\sum_{n}|\langle n|Q| 0\rangle|^{2}\left(\hbar \omega_{n}\right)^{k}
$$

where $Q$ is the transition operator. Bohigas et al. [67] have shown that an upper limit of the mean energy of a collective state is given in terms of the moments $m_{1}$ and $m_{3}$ by the energy $E_{3}$ defined as:

$$
E_{3}(Q)=\sqrt{\frac{m_{3}(Q)}{m_{1}(Q)}} .
$$

For pure surface oscillations in the direction of the symmetry axis $z$ and in the orthogonal direction $\varrho$, the excitation operators are the $z$ - and $\varrho$-components of the dipole operator $D$, respectively. For these simple operators, the moments $m_{1}$ and $m_{3}$ are particularly easy to evaluate and become [55]:

$$
\begin{aligned}
& E_{3}\left(D_{z}\right)^{2}=\left.\left(\frac{m_{3}}{m_{1}}\right)\right|_{z}=\frac{\hbar^{2}}{Z m} \int d^{3} r \rho_{e l}(\mathbf{r}) \frac{\partial^{2}}{\partial z^{2}} V_{J}(\mathbf{r}) \\
& E_{3}\left(D_{Q}\right)^{2}=\left.\left(\frac{m_{3}}{m_{1}}\right)\right|_{Q}=\frac{\hbar^{2}}{Z m} \int d^{3} r \rho_{e l}(\mathbf{r}) \frac{\partial^{2}}{\partial Q^{2}} V_{J}(\mathbf{r}) .
\end{aligned}
$$

The above relations are the so-called simple "sum rule estimates" of the peak positions of the dipole resonances. In the macroscopic limit, the energy $E_{3}$ obtained from the RPA moments $m_{1}$ and $m_{3}$ has been shown to go to the classical Mie frequency of the surface plasmon [55]. We are using these relations below to calculate the resonance energies in deformed clusters. In spherical clusters, we know that the excitation energies obtained in this approximation are too high by $10-15 \%$ due to the neglect 
Table 2 Energies of collective dipole resonances in the $E_{3}$ sum rule approximation (Values in brackets correspond to the isomers with higher energy)

\begin{tabular}{|c|c|c|c|c|c|}
\hline \multirow[t]{2}{*}{$Z$} & \multicolumn{2}{|c|}{ oblate isomer } & & \multicolumn{2}{|c|}{ prolate isomer } \\
\hline & $\hbar \omega_{z}[\mathrm{Ry}]$ & $\hbar \omega_{\perp}[\mathrm{Ry}]$ & & $\hbar \omega_{z}[\mathrm{Ry}]$ & $\hbar \omega_{\perp}[\mathrm{Ry}]$ \\
\hline 8 & & & 0.204 & & \\
\hline 10 & $(0.242)$ & $(0.203)$ & & 0.168 & 0.223 \\
\hline 12 & $(0.257)$ & $(0.205)$ & & 0.171 & 0.226 \\
\hline 14 & 0.254 & 0.186 & & $(0.174)$ & $(0.228)$ \\
\hline 16 & 0.242 & 0.197 & & $(0.192)$ & $(0.224)$ \\
\hline 18 & 0.234 & 0.205 & & - & - \\
\hline 20 & & & 0.216 & & \\
\hline 22 & $(0.229)$ & $(0.239)$ & & 0.194 & 0.224 \\
\hline 24 & $(0.238)$ & $(0.210)$ & & 0.190 & 0.239 \\
\hline 26 & $(0.246)$ & $(0.206)$ & & 0.184 & 0.238 \\
\hline 28 & $(0.250)$ & $(0.203)$ & & 0.191 & 0.239 \\
\hline 30 & $(0.251)$ & $(0.204)$ & & 0.192 & 0.231 \\
\hline 32 & 0.246 & 0.205 & & $(0.180)$ & $(0.240)$ \\
\hline 34 & 0.247 & 0.208 & & $(0.219)$ & $(0.225)$ \\
\hline 36 & $(0.236)$ & $(0.216)$ & & $(0.215)$ & $(0.228)$ \\
\hline 38 & 0.233 & 0.241 & & $(0.219)$ & $(0.223)$ \\
\hline 40 & & & 0.224 & & \\
\hline
\end{tabular}

Left columns: dipole mode along the $z$-axis;

Right columns: dipole mode in $\varrho$ direction (doubly degenerate).

of the coupling to the volume modes $[6,55,68]$. A more refined extension of these sumrule estimates, which allows to include the volume modes and their coupling to the surface modes, consists in the choice of a basis set of local excitation operators and solving the secular equation describing the coupled system of oscillators [55, 69]. This method can be based on a variational principle for the energy $E_{3}$, from which the RPA equations can be gained in principle, and thus has been termed the "local RPA" method $[6,50,68]$. The extension of the local RPA formalism to deformed clusters is in progress and will be studied in a forthcoming publication [70].

In Table 2 we present the results for the pure surface excitations $E_{3}$ for $\mathrm{Na}-8$ to $\mathrm{Na}-40$. In the triaxial case the dipole resonance splits into three equally weighted parts. If only axial deformations are involved, oscillations in the $\rho$ direction (perpendicular to the symmetry axis) become degenerate, having double weight compared to the excitation in the $z$ direction. Recent experimental evidence for a splitting of the main peak in $\mathrm{Na}-8$ and $\mathrm{Na}-20$ is beyond the simple local-RPA picture. In addition, although experimental evidence suggests that the dipole sum rule is widely exhausted, it has to be proven for each cluster that the collective excitation does not coincide with regions where the collective strength is fragmented by many different $1 \mathrm{p}-1 \mathrm{~h}$ transitions.

The systematics of surface dipole plasmons is shown in Fig. 15. Following Selby et al. [23], the plasmon peaks are artificially broadened

$$
\sigma_{o p t} \sim \sum_{\nu=z, \rho, \rho} \frac{(\hbar \omega)^{2} \Gamma_{v}}{\left((\hbar \omega)^{2}-\left(\hbar \omega_{\nu}\right)^{2}\right)^{2}+\left(\hbar \omega \Gamma_{\nu}\right)^{2}} .
$$




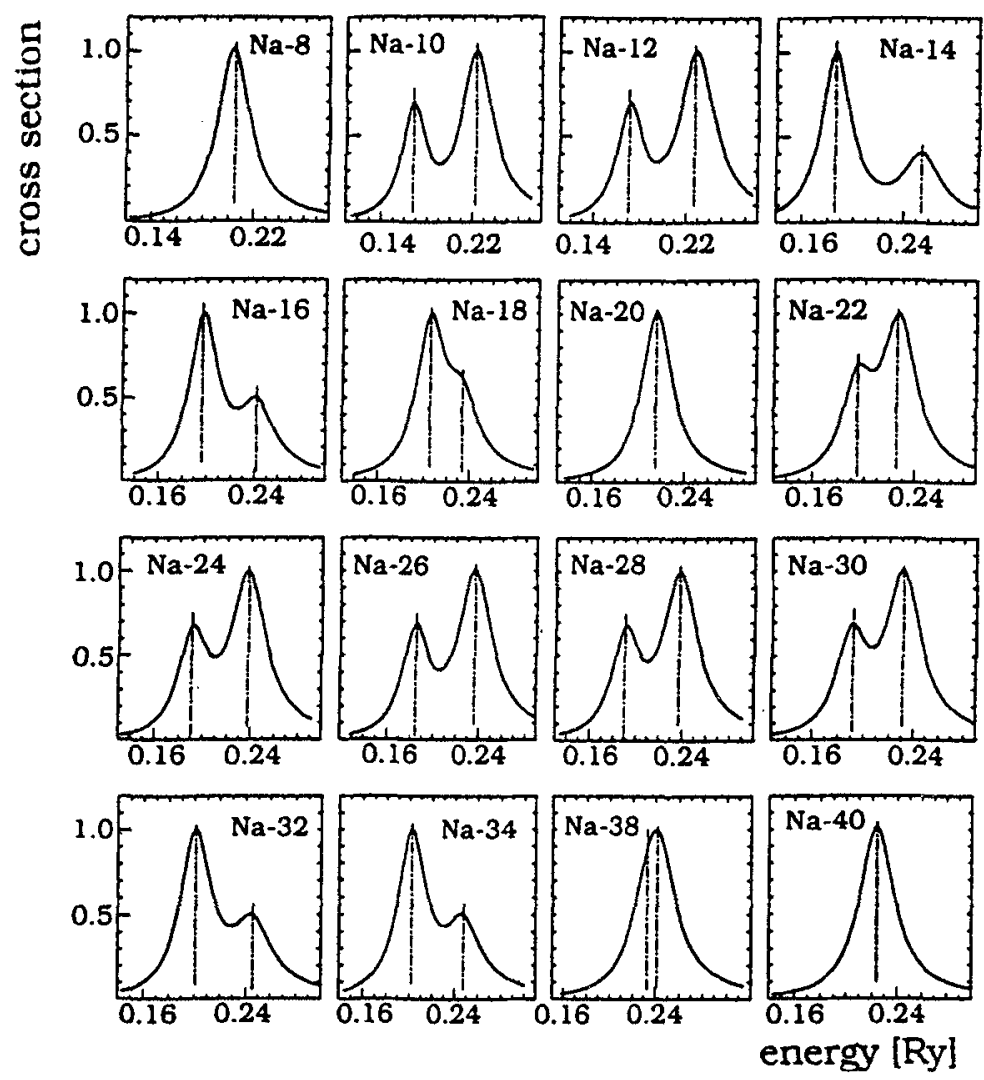

Fig. 15 Surface plasmons for $\mathrm{Na}-8$ to $\mathrm{Na}-40$. Transitions take place at particle number $12 / 14$, $18 / 20 / 22$ and $30 / 32$. As higher peaks are a result of a two-fold degeneracy of the modes in the $x / y$ plane, the oblate modes can easily be distinguished. Oblate clusters: $\hbar \omega_{\perp}<\hbar \omega_{z}$. Prolate clusters: $\hbar \omega_{\perp}>\hbar \omega_{2}$.

The phenomenological width $\Gamma_{v}=0.15 \hbar \omega_{v}$ illustrates the difficulty to resolve the plasmon peaks for clusters with small deformation. The third prolate-to-oblate transition at Na-30 lies very close to the experimental results. Near Na-28, Pedersen et al. [59] see some co-existing shapes. A close look at the potential energy curves Fig. 4 reveals that the differences in the ground state energies between oblate and prolate isomers have a minimum at $\mathrm{Na}-30$ and $\mathrm{Na}-32$. In these cases, the plasmon signals of oblate and prolate isomers have to be superposed. The plasmon plot of $\mathrm{Na}-36$ was omitted because $\mathrm{Na}-36$ does not follow the systematics within axial symmetry due to the reasons mentioned further above.

Although the cross sections shown in Fig. 15 are obtained in a highly schematic manner and their widths are put in by hand, they illustrate the close agreement between the deformation systematics obtained in our present static calculations and that found in the analysis of Pedersen et al. [59] of their experimental photoabsorption data.

\section{Conclusions}

Until now, the jellium model has remained the only tool for a systematic microscopic treatment of the valence electron system of metal clusters with $Z>20$. The agreement 
of its results with experimental data is encouraging, especially as far as the occurrence of peaks in cluster mass spectra and the deformation systematics extracted from the collective resonances in photoabsorption spectra is concerned.

However, the description of the cluster shape by spheroids or ellipsoids is too restrictive. As we have shown, higher than quadrupole multipolarities of the electronic density are important for the detailed balance between prolate and oblate shape isomers. Therefore, also in the description of the jellium density, these moments must be included in a more flexible way. This will be taken into consideration in future work. Giving up the left/right symmetry, i.e. including odd-parity multipolarities, should also be seriously considered in a systematic study of cluster shapes.

The neglect of the ionic structure is the most serious limitation of the simple jellium model. Ab-initio all-electron calculations by Bonačić-Koutecký et al. [71] have shown that the explicit treatment of the ionic structure leads to a much more quantitative description of the dipole response of small clusters with $N \leq 20$.

As a minimal phenomenological correction to reflect the ionic structure at least in the cluster surface, we have used a diffuse surface of the jellium density which is already well-known to improve the electronic dipole response $[49,50]$. As far as the static deformation properties are concerned which we have studied in the present work, we find that the diffuse jellium surface in most cases has little effect on the equilibrium deformations. As shown in Tab. 1, our ground-state deformations $\delta$ obtained in the mass region $8<N<40$ are practically identical, considering the overall uncertainties of the determination, with those of Ekardt and Penzar [24] who used the standard sharpsurface jellium model with spheroidal deformation. One exception is given by $\mathrm{Na}-34$, where a diffuse jellium surface has already earlier been observed to reduce the HOMOLUMO energy gap, thus shifting the situation in favour of the experimental situation, and where we find it here to lead to a somewhat larger oblate deformation than in the sharp-surface jellium model.

In order to establish a relation to the experimentally observed collective resonances ('plasmon peaks') in photoabsorption spectra, we have here used the most simple RPA sum-rule approach. However, collective excitation mechanisms can only be studied in detail using the single-particle wave functions obtained by selfconsistent calculations. This is a strong limitation of all schematic approaches, regardless how successful these models are in the correct description of the static shell structure. It has already been shown in spherical jellium model calculations that a considerable improvement over the simple surface plasmon model can be obtained in the time-dependent LDA (TDLDA) [64] and the random phase (RPA) [65, 72] schemes. Hartree-Fock plus RPA calculations by Guet et al. [73] within the jellium model confirm these results.

The only microscopic dipole response calculation in the deformed jellium model so far has been done by Ekardt and Penzar [74]; due to its numerical complexity, it was only done for one small cluster (Na-10). An approximate RPA treatment using localized collective currents ('local RPA') has been shown [68] to reproduce the leading moments of the fully microscopic RPA strength function of spherical clusters. The 'local RPA' scheme based on the selfconsistent deformed ground state densities obtained in the present model could therefore become an economic method for investigating the collective excitations of deformed clusters more quantitatively. 


\section{A Spheroidal deformation parameters}

With the definition of the quadrupole and hexadecupole operators $(n=\{2,4\})$

$$
\hat{Q}_{n}=r^{n} Y_{n 0}
$$

and the volume-conserved deformation $q=e^{\delta}$ of a spheroid

$$
\frac{z^{2}}{q^{4 / 3}}+\frac{\varrho^{2}}{q^{-2 / 3}}=R_{0}=r_{s} Z^{1 / 3}
$$

( $r_{s}$ being the Wigner-Seitz radius) the quadrupole and hexadecupole moments

$$
Q_{n 0}^{(j)}=\int d^{3} r \hat{Q}_{n} \rho_{J}(\mathbf{r})
$$

of the sharp-edged spheroid

$$
\rho_{J}(z, \varrho)=\left\{\begin{array}{l}
\rho_{0} \sqrt{q^{-4 / 3} z^{2}+q^{2 / 3} \varrho^{2}} \leq R_{0} \\
0 \sqrt{q^{-4 / 3} z^{2}+q^{2 / 3} \varrho^{2}}>R_{0}
\end{array}\right.
$$

can be calculated in cylindrical coordinates $(y, \varrho)$ :

$$
\begin{aligned}
& Q_{20}^{(j)}=\frac{2}{5} r_{s}^{2} Z^{5 / 3}\left(q^{4 / 3}-q^{-2 / 3}\right) \\
& Q_{40}^{(j)}=\frac{9}{70} \sqrt{\pi^{-1}} r_{s}^{4} Z^{7 / 3} q^{-4 / 3}\left(q^{2}-1\right)^{2} .
\end{aligned}
$$

The integration Eq. (31) cannot be performed analytically if the spheroid has a diffuse surface according to Eq. (13), but a simple substitution of the radial integration variable $r$ reveals that the deformation dependence of the radial part of the integral Eq. (31) can be put into a constant factor, such that the whole integral becomes independent of $q$. Fig. 2 gives a survey of the ratios of quadrupole moments for selected values of $\alpha$. The expansion coefficients of the radial harmonic expansion of the spheroid

$$
R(\theta)=R_{0}\left(1+b_{0}+\beta_{2} Y_{20}(\theta)+\beta_{4} Y_{40}(\theta)+\ldots\right)
$$

can be found inserting the exact angular dependency of the radius of the spheroid

$$
r(\theta)=\frac{R_{0} q^{2 / 3}}{\sqrt{\left(1-q^{2}\right) \cos ^{2} \theta+q^{2}}}
$$

into the projection

$$
\beta_{n}=\frac{1}{R_{0}} \int d \Omega r(\theta) Y_{n 0}(\Omega)
$$


$b_{0}$, which is defined in [53], ensures that the volume is conserved. Again we obtain analytical expressions for the expansion coefficients $\beta_{2}$ and $\beta_{4}$

$$
\beta_{2}^{(j)}=\sqrt{\frac{5 \pi}{4}} \frac{q^{2 / 3}}{1-q^{2}} \begin{cases}3-\frac{q^{2}+2}{\sqrt{1-q^{2}}} \operatorname{arccosh}\left(q^{-1}\right) & \text { if } q<1 \\ 3-\frac{q^{2}+2}{\sqrt{q^{2}-1}} \arccos \left(q^{-1}\right) & \text { if } q>1\end{cases}
$$

and

$$
\beta_{4}^{(j)}=\frac{3}{64} \sqrt{\pi} \frac{q^{2 / 3}}{\left(1-q^{2}\right)^{2}}\left(3\left(3 q^{4}+24 q^{2}+8\right) \mathscr{F}-10\left(11 q^{2}+10\right)\right)
$$

with

$$
\mathscr{F}= \begin{cases}\frac{2}{\sqrt{1-q^{2}}} \operatorname{arccosh}\left(q^{-1}\right) & q<1 \\ \frac{2}{\sqrt{q^{2}-1}} \arccos \left(q^{-1}\right) & q>1 .\end{cases}
$$

Note that $\beta_{2}$ is different from the definition in Ref. [22]. If one only considers the volume and quadrupole term in the expansion Eq. (13), the axis ratio is given by [60]:

$$
\beta_{2}^{(j)}=\sqrt{\frac{16 \pi}{5}}(q+2)^{-1} \begin{cases}(q-1) & \text { if } q>1 \\ (1-q) & \text { if } q<1\end{cases}
$$

This partly explains the small discrepancy in the position of the equilibrium deformations of $\mathrm{Na}-12$ and $\mathrm{Na}-14$ in [22].

\section{B Technical remarks}

In order to calculate wave functions and matrix elements of a deformed cluster, we introduce oscillator constants

$$
\beta_{z}=\sqrt{\frac{m \omega_{z}}{\hbar}} \text { and } \beta_{\perp}=\sqrt{\frac{m \omega_{\perp}}{\hbar}}
$$

and transform onto a mesh with dimensionless coordinates

$$
\xi=z \beta_{0} q^{-1 / 3} \text { and } \eta=\varrho^{2} \beta_{0}^{2} q^{1 / 3}, \text { with } \beta_{0}=\sqrt{\frac{m \omega_{0}}{\hbar}} \text {. }
$$

Note that the level spectrum of the axially deformed harmonic oscillator scales with the deformation parameter $q_{b}$ defined by 


$$
q_{b}=\frac{\omega_{\perp}}{\omega_{z}} .
$$

Choosing this scaling procedure, volume conservation is approximately preserved by construction. For the deformation parameter of the basis system we chose $q_{b}=q$, which allows an optimized selection of the basis levels within a deformation-dependent cut-off in the expansion Eq. (20), e.g., for prolate configurations levels with large $n_{z}$ and small $n_{r}$ and $|\Lambda|$ have been selected, whereas the total number of levels was fixed.

Selfconsistent calculations of deformed Coulomb systems still demand some effort with regard to numerical details. On the one hand, long range contributions of the nonlocal Hartree term can cause severe errors if the mesh size is chosen too small. As we already know from special calculations, there are large cancellations of the Coulomb contributions due to the spherical features of the jellium model, which demand relatively high numerical precision in the calculation of the Coulomb interaction. In fact, we had to extend the mesh size until the condition

$$
\int \Delta \rho(\mathbf{r}) d^{3} r=0
$$

was approximately satisfied. Another criterion for the precision of the Coulomb integration is given by

$$
\int V_{J}(\mathbf{r}) \rho_{e}(\mathbf{r}) d^{3} r=\int V_{e}(\mathbf{r}) \rho_{J}(\mathbf{r}) d^{3} r .
$$

Most of the binding is attributed to exchange and correlation effects which are small in magnitude compared with e.g. the electron-jellium interaction. Thus the Coulomb integrations have to be performed with care. On the other hand, computation time for the Hartree and jellium interaction parts increases rapidly if the mesh is enlarged. Calculations have been performed with a total number of main shells $N_{0}=12$ for $\mathrm{Na}-8$ to Na-20 on a $32 \times 32$ mesh, $N_{0}=14$ for Na-22 to Na-34 on an $64 \times 64$ mesh, and up to $\mathrm{Na}-40$ for $N_{0}=16$ on the same mesh.

As all integrals have been calculated using Gauss-Hermite and Gauss-Laguerre quadrature formulas according to [54], any extension of the mesh is inevitably linked with an increase of the number of mesh points. Thus the calculation of the Hartree contributions in coordinate space becomes inefficient as soon as it takes more time as the diagonalization. On a $64 \times 64$ mesh, as it was used for clusters $Z>20$, one obtains a mean step width of 0.80 to $1.00 \mathrm{a}$.u.

This seems to be a large value at first sight, but one has to consider that all first and second derivatives are given analytically due to the fact that all quantities are expanded into an analytically known basis.

The inverse oscillator length $\beta_{0}$ had to be adjusted in order to enhance convergence and minimize the total energy. From Fig. 16 we learn that the total energy depends on the choice of $\beta_{0}$, if $\beta_{0}$ is chosen inadequately. A good plateau condition for larger clusters with $Z \geq 34$ was obtained with $N_{0}=16$. As the parameter $\beta_{0}$ scales the basis function system used on the mesh, one would obtain an additional criterion for the choice of the mesh size.

The basis truncation effect is visualized in Fig. 17. Na-36 is predicted triaxial because of the near-degeneracy of the total energy and the strong mixing of basis levels. This 
Fig. 16 Na-36: Stationarity of the total energy with regard to the oscillator parameter is required. For larger clusters like $\mathrm{Na}-36$, this condition is satisfied for $N_{0}=16$ and $\beta_{0} \sim 0.23$.

Fig. 17 Total energy of Na-36. Influence of $N_{0}$ and the oscillator parameter $\beta_{0}$ on $E_{\text {tot }}$. The number of principal shells $N_{0}$ has been increased until the difference to spherical calculations became negligible at $\delta=0$. Mesh size effects become important if the inverse oscillator length $\beta_{0}$ is adjusted to small values.
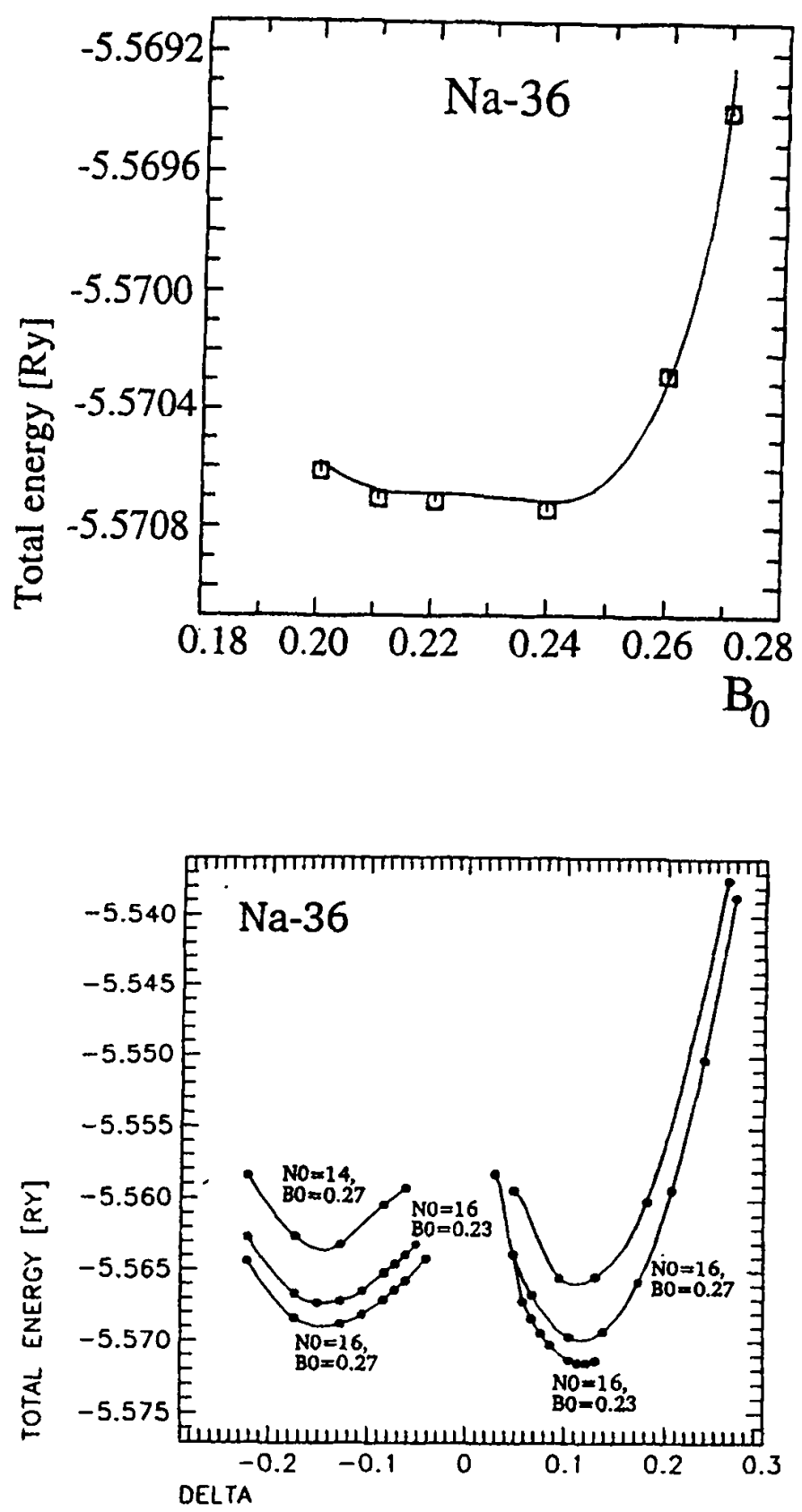

cluster appears to be a good touchstone for the choice of parameters, because the results were very sensitive to truncation effects and the choice of parameters.

We obtained a very sharp criterion for the convergence of our results by inserting the spherical Kohn-Sham results into the PES curves in Fig. 4. The results are obviously very well converged, as the curves fit smoothly through the spherical points. 
Extensive discussions with P.-G. Reinhard, S.M. Reimann, W. Seidl and B. Montag have been valuable contributions to this work. We would like to thank $H$. Bresele for his help in the spherical calculations. This work has been partially supported by the Commission of the European Communities under SCIENCE Contract No. ERB4002PL-910205.

\section{References}

[1] M. Cini, J. Catal. 37 (1975) 187

[2] A. Hintermann, M. Manninen, Phys. Rev. B 27 (1983) 7262

[3] W. Ekardt, Phys. Rev. B 29, 4 (1984) 1558

[4] D. E. Beck, Phys. Rev. B 30 (1984) 6935

[5] W.A. de Heer, Rev. Mod. Phys. 65 (1993) 611

[6] M. Brack, Rev. Mod. Phys. 65 (1993) 677

[7] K. Clemenger, Phys. Rev. B 32 (1985) 1359

[8] W.A. Saunders, Ph. D. thesis, University of California, Berkeley, unpublished (1986)

[9] H. Nishioka, K. Hansen, B. R. Mottelson, Phys. Rev, B 42 (1990) 9377

[10] J. Pedersen, S. Bjørnholm, J. Borggreen, J. Hansen, T. P. Martin, H.D. Rasmussen, Nature 353 (1991) 733

[11] T.P. Martin, S. Bjørnholm, J. Borggreen, C. Bréchignac, P. Cahuzac, K. Hansen, J. Pedersen, Chem. Phys. Lett. 186 (1991) 53

[12] C. Bréchignac, P. Cahuzac, M. de Frutos, J.P. Roux, K. H. Bowen, in: Physics and Chemistry of finite systems: from clusters to crystals, $P$. Jena et al. (ed.), Kluwer Academic Publishers, Dordrecht, Vol. I, 1992, p. 369

[13] O. Genzken, M. Brack, Phys. Rev. Lett. 67 (1991) 3286

[14] R. Balian, C. Bloch, Ann. Phys. (N.Y.) 69, 76; and earlier references quoted therein (1972)

[15] V. Bonačić-Koutecký, P. Fantucci, J. Koutecký, Chem. Rev. 91 (1991) 1035

[16] J. Gally, M. Parrinello, in: Computer simulation in material science, NATO ASI Series E: 'Applied Sciences', Vol. 205, M. Meyer, V. Pontikis (eds.) Kluwer Academic Publ., Dordrecht (1991), p. 283

[17] U. Röthlisberger, W. Andreoni, J. Chem. Phys. 94 (12) (1991) 8129

[18] W.D. Knight, K. Clemenger, W. A. de Heer, W. A. Saunders, M. Y. Chou, M. L. Cohen, Phys. Rev. Lett. 52 (1984) 2141

[19] S. G. Nilsson, Mat. Fys. Medd. Dan. Vid. Selsk. 29 (1955) no. 16

[20] H.A. Jahn, E. Teller, Proc. Roy. Soc. (London) A 161 (1937) 220

[21] W. Ekardt, Z. Penzar, Phys. Rev. B 38 (1988) 4273

[22] G. Lauritsch, P.-G. Reinhard, J. Meyer, M. Brack, Phys. Lett. A 160 (1991) 179

[23] K. Selby, M. Vollmer, J. Masui, V. Kresin, W.A. de Heer, W.D. Knight, Phys. Rev. B 40, No. 8 (1989) 5417

[24] Z. Penzar, W. Ekardt, Z. Phys. D 17 (1990) 69

[25] Th. Hirschmann, M. Brack, J. Meyer, Computational Materials Science 2 (1994) in press

[26] O. Gunnarsson, B.I. Lundqvist, Phys. Rev. B 13, No. 10 (1976) 4274

[27] J.P. Perdew, A. Zunger, Phys. Rev, B 23 (1981) 5048

[28] J.A. Alonso, L. A. Girifalco, Sol. State Comm. 24 (1977) 135

[29] O. Gunnarsson, M. Jonson, B. I. Lundqvist, Sol. State Comm. 24 (1977) 765

[30] R.M. Dreizler, E.K.U. Gross, Density Functional Theory, Springer-Verlag, Berlin, 1990

[31] E.K.U. Gross, W. Kohn, Adv, in Quant. Chem. 21 (1990) 255

[32] P. Stampfli, K. H. Bennemann, in: Physics and Chemistry of Small Clusters, P. Jena, B. K. Rao, S. N. Khanna (eds.) Plenum, New York (1987) p. 473

[33] S. Saito, S. B. Zhang, S. G. Louie, M.L. Cohen, J. Phys.: Condens. Matter 2 (1990) 9041

[34] J. M. Pacheco, W. Ekardt, Z. Phys. D 24 (1992) 65; Ann. Phys. (Leipzig) 1 (1992) 254

[35] L. C. Balbás, J.A. Alonso, A. Rubio, Europhys. Lett. 14 (1991) 323

[36] A. Rubio, L.C. Balbás, J. A. Alonso, Physica B 168 (1991) 32

[37] C. Guet, W. R. Johnson, M. Madjet, Z. Phys. D 26 (1993) 125

[38] P.-G. Reinhard, Phys. Lett. A 169 (1992) 281

[39] W. Kohn, L. J. Sham, Phys. Rev. A 140, No. 4 (1965) A 1133 
[40] R. Car, M. Parrinello, Phys. Rev. Lett. 55 (1985) 2471

[41] C. A. Utreras-Díaz, H. B. Shore, Phys. Rev. Lett. 24 (1984) 2335

[42] C. A. Utreras-Díaz, H. B. Shore, Phys. Rev. B 40 (1989) 10345

[43] J.P. Perdew, H.Q. Tran, E.D. Smith, Phys. Rev. B 42 (1990) 11627

[44] M. Brajczewska, C. Fiolhais, J. P. Perdew, Int. J. of Quantum Chemistry 1993, in press

[45] B. Montag, P.-G. Reinhard, J. Meyer, Z. Phys. D (1994) in print

[46] M.P. Iñiguez, M.J. Lopez, J.A. Alonso, J. M. Soler, Z. Phys. D 11 (1989) 163

[47] G. Borste!, U. Lammers, A. Mañanes, J.A. Alonso, in: Nuclear Physics Concepts in Atomic Cluster Physics, R. Schmidt, H. O. Lutz, R. Dreizler (eds.) Springer-Verlag, Berlin, 1992, p. 327

[48] B. Montag, P.-G. Reinhard, J. Meyer, Th. Hirschmann, M. Brack, to be published

[49] L. C. Balbás, A. Rubio, Anal. Fis. (Spain) A 86 (1990) 207; A. Rubio, L. C. Balbás, J. A. Alonso, Z. Phys. D 19 (1991) 93

[50] P.-G. Reinhard, S. Weisgerber, O. Genzken, M. Brack, in: Nuclear Physics Concepts in Atomic Cluster Physics, R. Schmidt, H. O. Lutz, R. Dreizler (eds.) Springer-Verlag, Berlin, 1992, p. 254

[51] Ashcroft, N.W., J. Phys. C (Proc. Phys. Soc.), Ser. 2, Vol. 1 (1967) 233

[52] S. M. Reimann, M. Brack, K. Hansen, Z. Phys. D 28 (1993) 235

[53] M. Brack, T. Ledergerber, H.C. Pauli, Nucl. Phys. A 234 (1974) 185-215

[54] D. Vautherin, Phys. Rev. C 7, No. 1 (1973) 296

[55] M. Brack, Phys. Rev. B 39 (1989) 3533

[56] S. Bjørnholm, J. Borggreen, O. Echt, K. Hansen, J. Pedersen, H. D. Rasmussen, Phys. Rev. Lett. 65 (1990) 1727

[57] P.-G. Reinhard, private communication (1992), using the code of Lauritsch et al., Ref. [22]

[58] S. Frauendorf, V. V. Pashkevich, Z. Phys. D 26 (1993) S-98

[59] J. Borggreen, P. Chowdhury, N. Kabaili, L. Lundsberg-Nielsen, K. Lützenkirchen, M. B. Nielsen, J. Pedersen, H.D. Rasmussen, Phys. Rev. B 48 (1993) 17507

[60] P. Ring, P. Schuck, in: The Nuclear Many-Body Problem, Springer 1980

[61] I. Hamamoto, B. R. Mottelson, H. Xie, X.Z. Zhang, Z. Phys. D 21 (1991) 163

[62] M. Danos, Nucl. Phys. 5 (1958) 23

[63] M. Goldhaber, E. Teller, Phys. Rev. 74 (1948) 1046

[64] W. Ekardt, Phys. Rev. B 31, 6360; W. Ekardt, Phys. Rev. B 32 (1985) 1961

[65] C. Yannouleas, R. A. Broglia, M. Brack, P.F. Bortignon, Phys. Rev. Lett. 63 (1989) 255

[66] C. Yannouleas, R. A. Broglia, Phys. Rev. A 44 (1991) 5793

[67] O. Bohigas, A.M. Lane, J. Martorell, Phys. Rep. 51, No. 5 (1979) 5269

[68] P.-G. Reinhard, M. Brack, O. Genzken, Phys. Rev. A 41, No. 10 (1990) 5568

[69] P. Gleissl, M. Brack, J. Meyer, P. Quentin, Annals of Physics (N.Y.), Academic Press (1990) 205

[70] Th. Hirschmann, M. Brack, to be published

[71] V. Bonacick-Koutecký, C. Fuchs, J. Pittner, J. Koutecký, P. Fantucci, 1st International Conference on the Theory of Atomic and Molecular Clusters, Leer 1993, Germany

[72] C. Yannouleas, E. Vigezzi, R. A. Broglia, Preprint NBI-92-54 (1992)

[73] C. Guet, W. R. Johnson, Phys. Rev. B 45 (1992) 11283

[74] W. Ekardt, Z. Penzar, Phys. Rev. B 43 (1991) 1322 